\title{
Efficient discovery of SARS-CoV-2-neutralizing antibodies via B cell receptor sequencing and ligand blocking
}

\author{
Andrea R. Shiakolas ${ }^{1,2}$, Kevin J. Kramer ${ }^{10}{ }^{1,2,12}$, Nicole V. Johnson ${ }^{\circledR 3,12}$, Steven C. Wall ${ }^{1,2,12}$, \\ Naveenchandra Suryadevara $\mathbb{D}^{1}$, Daniel Wrapp ${ }^{3}{ }^{3}$, Sivakumar Periasamy ${ }^{4,5}$, Kelsey A. Pilewski ${ }^{1,2}$, \\ Nagarajan Raju ${ }^{1,2}$, Rachel Nargi', Rachel E. Sutton', Lauren M. Walker ${ }^{1,2}$, Ian Setliff ${ }^{1}$, \\ James E. Crowe Jr(1,2,6, Alexander Bukreyev ${ }^{4,5,7}$, Robert H. Carnahan ${ }^{1,6}$, Jason S. McLellan $\mathbb{1}^{3}$ \\ and Ivelin S. Georgiev 1, 1,2,8,9,10,11凶
}

\begin{abstract}
Although several monoclonal antibodies (mAbs) targeting severe acute respiratory syndrome coronavirus 2 (SARS-CoV-2) have been approved for coronavirus disease 2019 (COVID-19) therapy, development was generally inefficient, with lead generation often requiring the production and testing of numerous antibody candidates. Here, we report that the integration of target-ligand blocking with a previously described B cell receptor-sequencing approach (linking B cell receptor to antigen specificity through sequencing (LIBRA-seq)) enables the rapid and efficient identification of multiple neutralizing $\mathrm{mAbs}$ that prevent the binding of SARS-CoV-2 spike (S) protein to angiotensin-converting enzyme 2 (ACE2). The combination of target-ligand blocking and high-throughput antibody sequencing promises to increase the throughput of programs aimed at discovering new neutralizing antibodies.
\end{abstract}

T echnologies for developing preventive and therapeutic measures that can counteract potential pandemics are of the utmost significance for public health. The COVID-19 pandemic has emphasized the importance of rapid countermeasure development. Through pandemic preparedness initiatives, effective SARS-CoV-2-neutralizing antibodies were discovered and validated within months ${ }^{1-6}$, as were SARS-CoV-2 vaccine candidates ${ }^{7}$. However, even with such unprecedented speed of vaccine and therapeutic development, the pandemic has inflicted devastating worldwide effects. Accelerating actions by weeks or months can make an enormous difference in an exponentially evolving pandemic. Therefore, efficient methods for discovery of effective countermeasures against emerging pathogens can play a critical role in pandemic preparedness for future infectious disease outbreaks.

Antibodies are a major modality for therapy and vaccine design strategies for a wide range of diseases; however, the functional antibody discovery process can be inefficient. Typically, at the screening step, B cells are prioritized based on antigen recognition, but this often requires time-intensive subsequent $\mathrm{mAb}$ validation steps for discovery of functional neutralizing antibodies. This limitation was exemplified by SARS-CoV-2 antibody discovery initiatives, as testing of large numbers of antibodies (frequently hundreds to thousands) was generally required to identify a small fraction of neutralizing antibodies with a wide range of hit rates when using S protein as an antigen bait (about 2 to $23 \%$ ) or when using receptor-binding domain (RBD) and/or S protein subunit 1 (S1) (about $2-55 \%)^{1-6,8-12}$ in various studies.

\section{Results}

To overcome this limitation, we developed LIBRA-seq with ligand blocking, a second-generation LIBRA-seq technology that incorporates a functional readout into the antibody discovery process ${ }^{13}$. LIBRA-seq uses DNA-barcoded antigens to map antibody sequence to antigen specificity using next-generation sequencing ${ }^{13}$. For LIBRA-seq with ligand blocking, a ligand and its cognate target antigen(s) are each labeled with a unique oligonucleotide barcode (Extended Data Fig. 1a), enabling the transformation of antigenligand interactions into sequenceable events. In these experiments, $\mathrm{B}$ cells that can block antigen-ligand interactions are expected to have high LIBRA-seq scores for the target antigen(s) and low LIBRA-seq scores for the ligand (Extended Data Fig. 1a). Therefore, a single high-throughput LIBRA-seq with ligand blocking experiment provides both antigen recognition and ligand-blocking information simultaneously for many B cells.

To evaluate this technology, we sought to discover SARS-CoV2-specific antibodies from $B$ cells from individuals with past SARS-CoV-2 infection, because antibodies that block the interactions between the SARS-CoV-2 S protein and its host receptor ACE2 are among the most potently neutralizing identified

'Vanderbilt Vaccine Center, Vanderbilt University Medical Center, Nashville, TN, USA. ${ }^{2}$ Department of Pathology, Microbiology, and Immunology, Vanderbilt University Medical Center, Nashville, TN, USA. ${ }^{3}$ Department of Molecular Biosciences, The University of Texas at Austin, Austin, TX, USA. ${ }^{4}$ Department of Pathology, University of Texas Medical Branch at Galveston, Galveston, TX, USA. ${ }^{5}$ Galveston National Laboratory, University of Texas Medical Branch at Galveston, Galveston, TX, USA. ${ }^{6}$ Department of Pediatrics, Vanderbilt University Medical Center, Nashville, TN, USA. ${ }^{7}$ Department of Microbiology and Immunology, University of Texas Medical Branch at Galveston, Galveston, TX, USA. ${ }^{8}$ Vanderbilt Institute for Infection, Immunology, and Inflammation, Vanderbilt University Medical Center, Nashville, TN, USA. ${ }^{9}$ Department of Computer Science, Vanderbilt University, Nashville, TN, USA. ${ }^{10} \mathrm{Center}$ for Structural Biology, Vanderbilt University, Nashville, TN, USA. "1Program in Computational Microbiology and Immunology, Vanderbilt University Medical Center, Nashville, TN, USA. ${ }^{12}$ These authors contributed equally: Kevin J. Kramer, Nicole V. Johnson, Steven C. Wall. 凶e-mail: ivelin.georgiev@vanderbilt.edu 
Experiment 1: ligand blocking

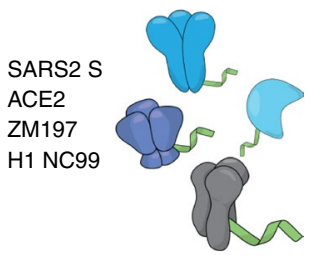

b

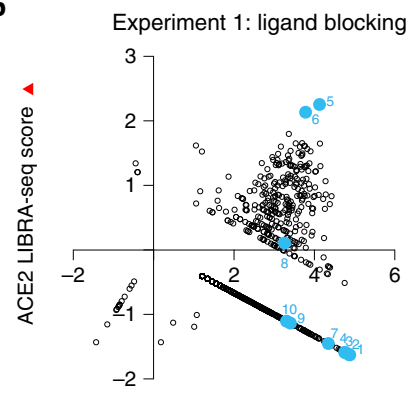

SARS-CoV-2 S LIBRA-seq score

Experiment 2: SARS-CoV-2 S titration

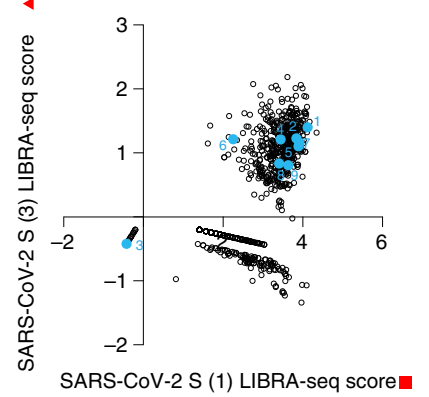

d

Experiment 3: SARS-CoV-2 S titration and ligand blocking

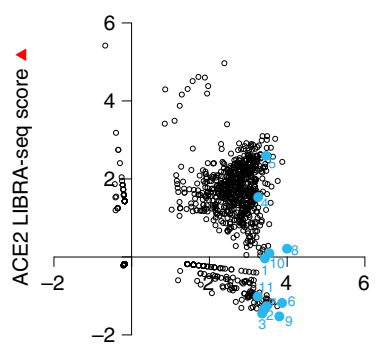

SARS-CoV-2 S (1) LIBRA-seq score
Experiment 2: SARS-CoV-2 S titration

Experiment 3: SARS-CoV-2 S titration and ligand blocking
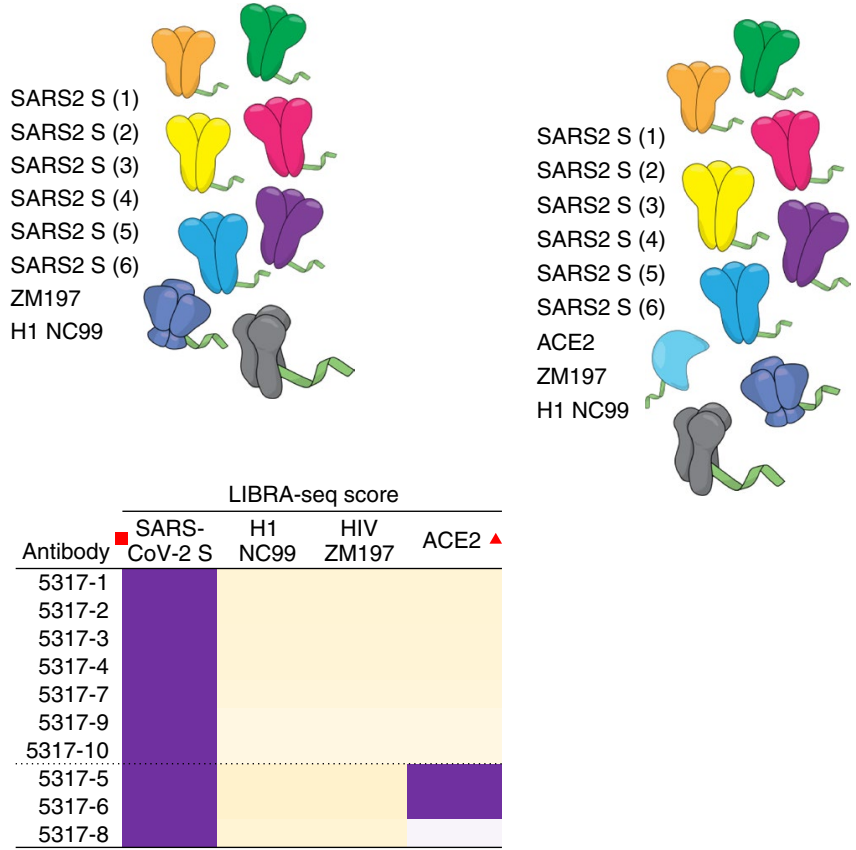

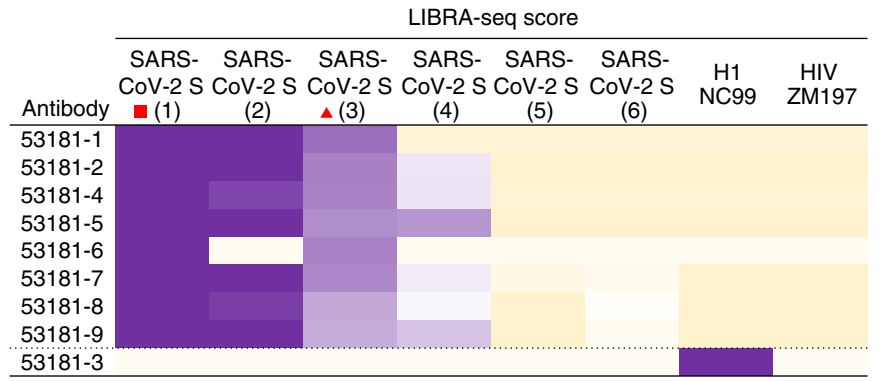

$53181-3$

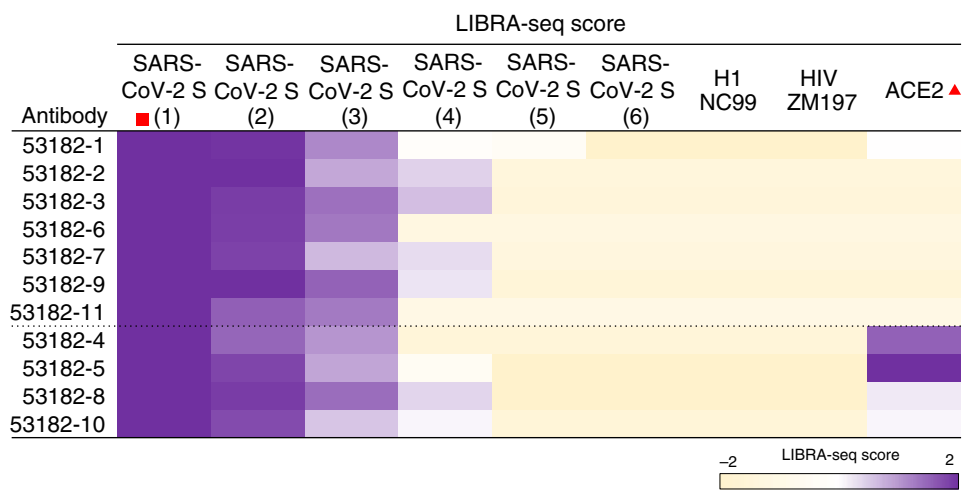

Fig. 1 | Antibody discovery using LIBRA-seq with ligand blocking. a, Experimental setup of three LIBRA-seq experiments: experiment 1, LIBRA-seq with ligand blocking; experiment 2, LIBRA-seq with a SARS-CoV-2 S (SARS2 S) titration; experiment 3, LIBRA-seq with a SARS-CoV-2 S titration and ligand blocking. For experiments 2 and 3, six different aliquots of S protein were added in a titration series (1-6); HIV ZM197 envelope and influenza hemagglutinin H1 NC99 were used as controls. b-d, Left, after next-generation sequencing, hundreds of B cells (dots) were recovered that had paired heavy/light chain sequencing information and antigen reactivity information for the three experiments. For experiments 1 (b), 2 (c) and 3 (d), select LIBRA-seq scores for all cells per experiment are shown as open circles ( $n=828,829$ and 957 , respectively). Antibodies selected for expression and validation are highlighted and numbered in light blue. Right, LIBRA-seq scores for the selected antibodies for all antigens from each experiment are shown as a heat map from $-2(\tan )$ to 2 (purple); scores outside of this range are shown as the minimum and maximum values. For experiments 1 and 3 , antibodies with negative scores for ACE2 are shown above the dotted line, while antibodies with positive scores for ACE2 are shown below the dotted line and are controls. For experiment 2, all SARS-CoV-2-reactive antibodies are shown above the dotted line, whereas influenza-specific antibody 53181-3 is shown as a control below the dotted line. 
a

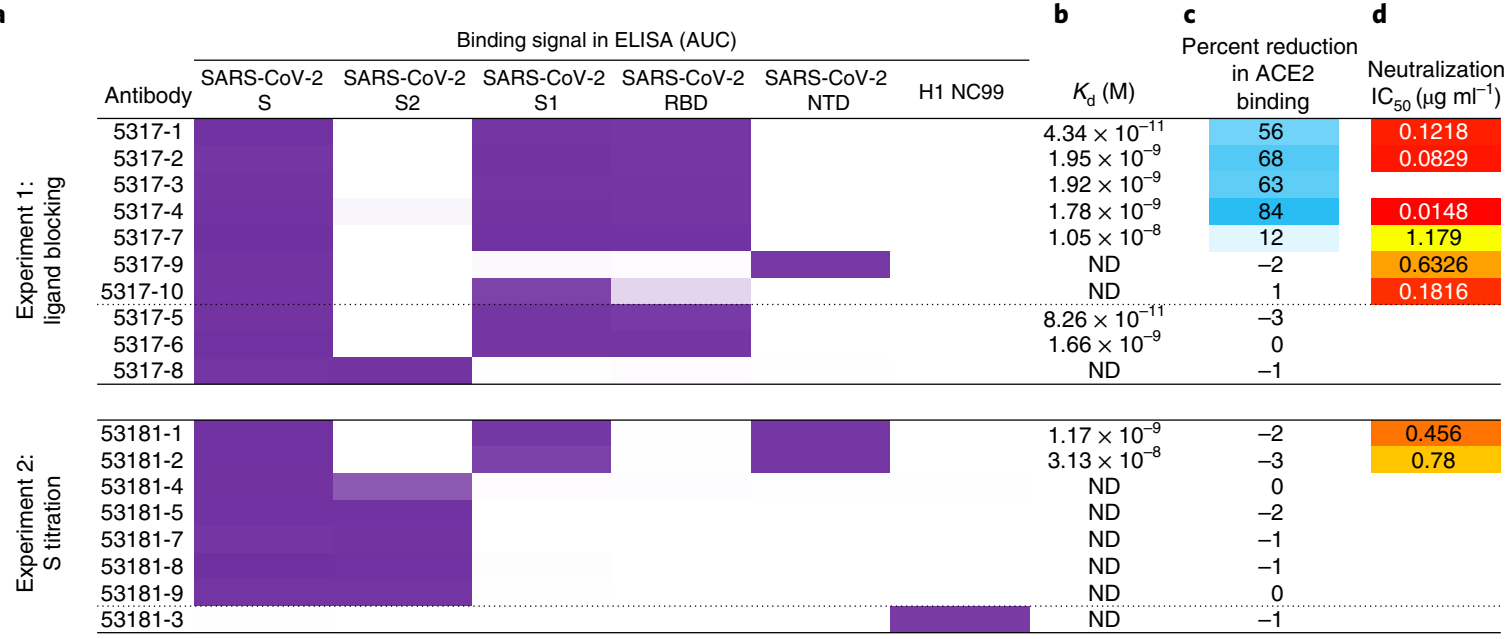

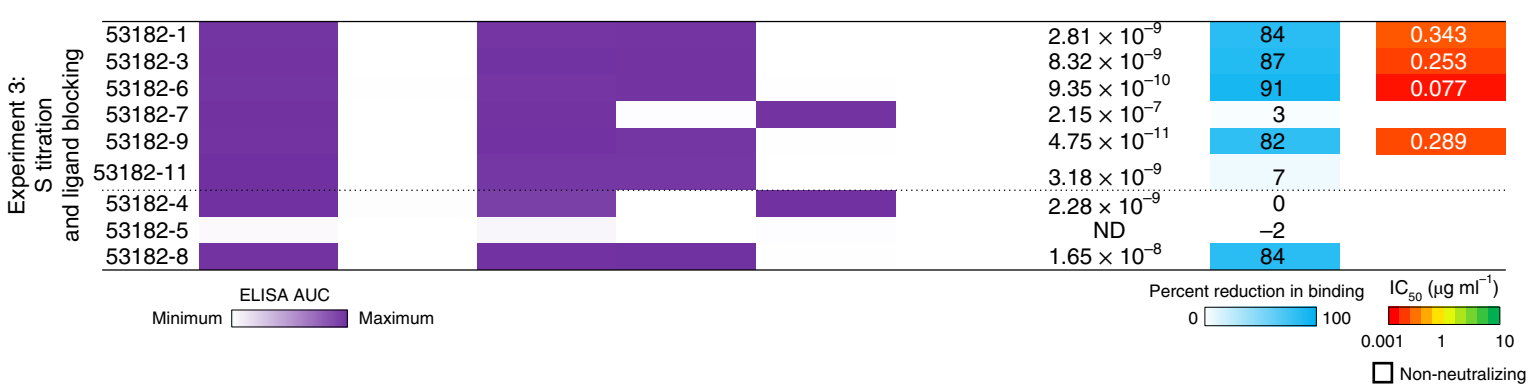

Fig. 2 | Validation and characterization of selected antibodies. a, ELISA area under the curve (AUC) values for binding to SARS-CoV-2 recombinant antigen proteins and a negative control influenza hemagglutinin protein are shown for antibodies (rows) in each experiment and were calculated from data in Extended Data Fig. 2b. b, $K_{\mathrm{d}}(\mathrm{M})$ values of antibodies for SARS-CoV-2 RBD or NTD (based on epitope shown in a) were determined by biolayer interferometry; ND, not done. c, Percent reduction in ACE2 binding, as measured by ELISA, is shown as a heat map from 0 to $100 \%$ (white to blue) reduction in binding compared to SARS-CoV-2 binding only. $\mathbf{d}$, Vesicular stomatitis virus (VSV)-SARS-CoV-2 neutralization half-maximum inhibitory concentration $\left(I_{50}\right)$ values are shown as a heat map from high potency (red) to low potency (green). Non-neutralizing antibodies are shown as white.
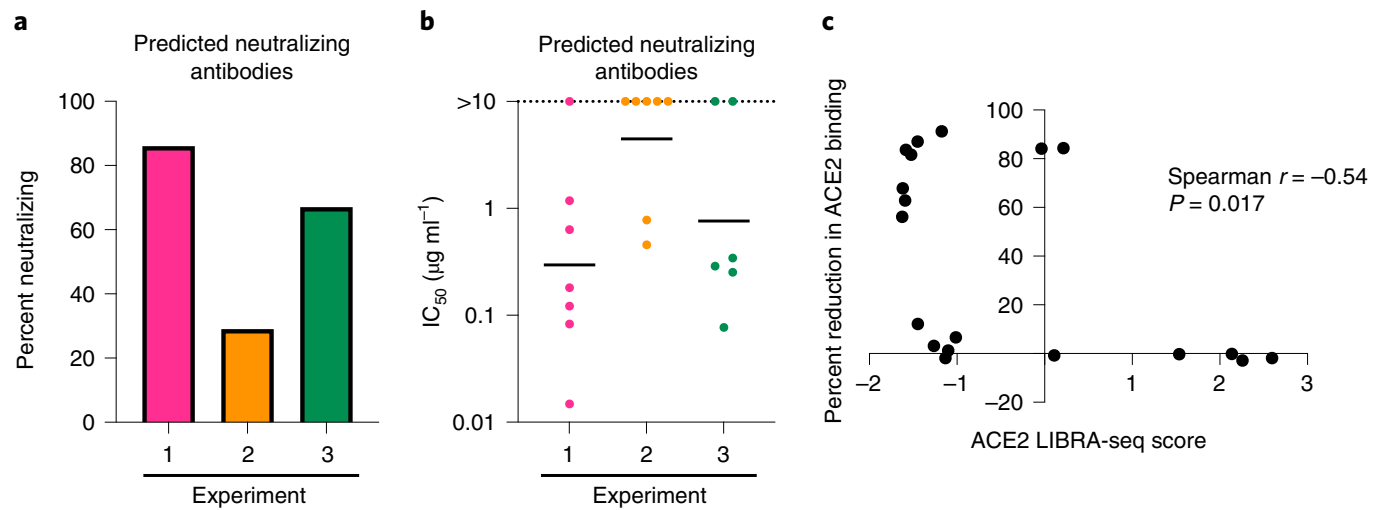

Fig. 3 | Assessment of LIBRA-seq with ligand blocking. a, Predicted neutralizing antibodies were defined as the subset of selected antibodies with negative ACE2 LIBRA-seq scores from experiments 1 ( $n=7$ antibodies) and 3 ( $n=6$ antibodies) and all antibodies with high LIBRA-seq Scores ( $>1)$ for SARS-CoV-2 $\mathrm{S}$ from experiment 2 ( $n=7$ antibodies). The percentage of neutralizing antibodies from the set of predicted neutralizers is shown for each

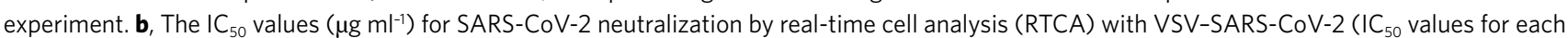
antibody are shown as single dots) are plotted for the set of predicted neutralizers. The horizontal lines represent the geometric mean for each experiment. Non-neutralizing antibodies are shown as $>10 \mu \mathrm{ml}^{-1}$. c, Spearman correlation of ACE2 LIBRA-seq score ( $x$ axis) and percent reduction in ACE2 binding to SARS-CoV-2 ( $y$ axis) for antibodies from experiments 1 and 3; Spearman $r=-0.54, P=0.017$ (two tailed, $95 \%$ confidence interval).

to date $e^{2-6,8,9}$. We performed three LIBRA-seq experiments with screening libraries that included the following: experiment 1, ACE2 and SARS-CoV-2 S; experiment 2, a titration series of different aliquots of SARS-CoV-2 S, each labeled with a unique barcode; and experiment 3, ACE2 and a titration series of S (Fig. 1a). The incorporation of a titration series of $\mathrm{S}$ antigen in the screening library for experiments 2 and 3 aimed to assess the strength of B cell receptorantigen interactions (Extended Data Fig. 1b,c). 

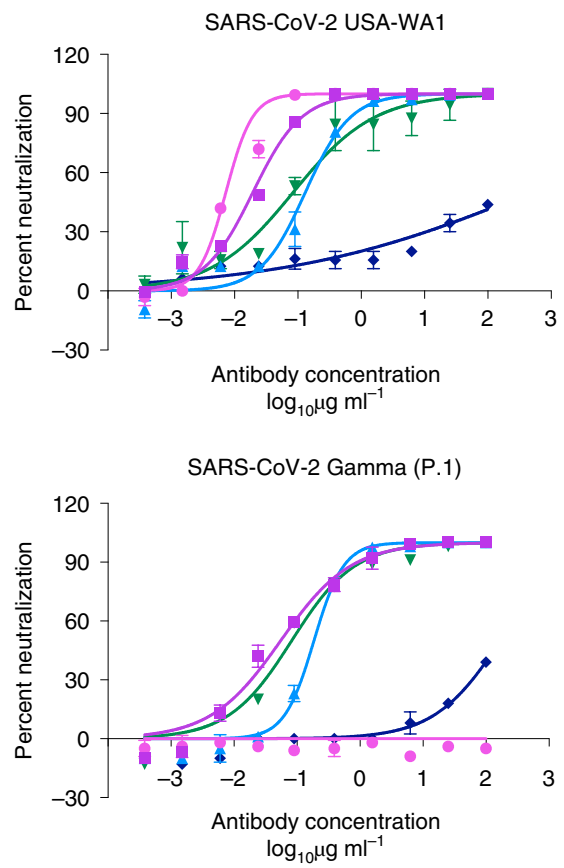

SARS-CoV-2 Alpha (B.1.1.7)

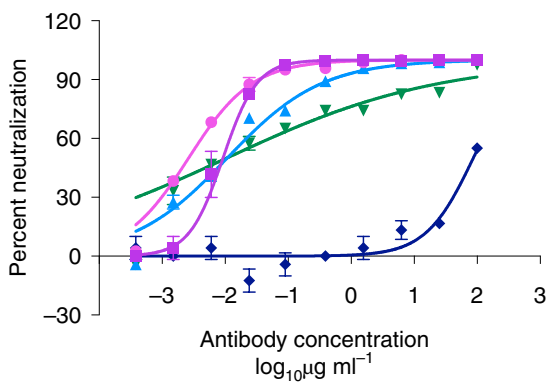

SARS-CoV-2 Delta (B.1.617.2)

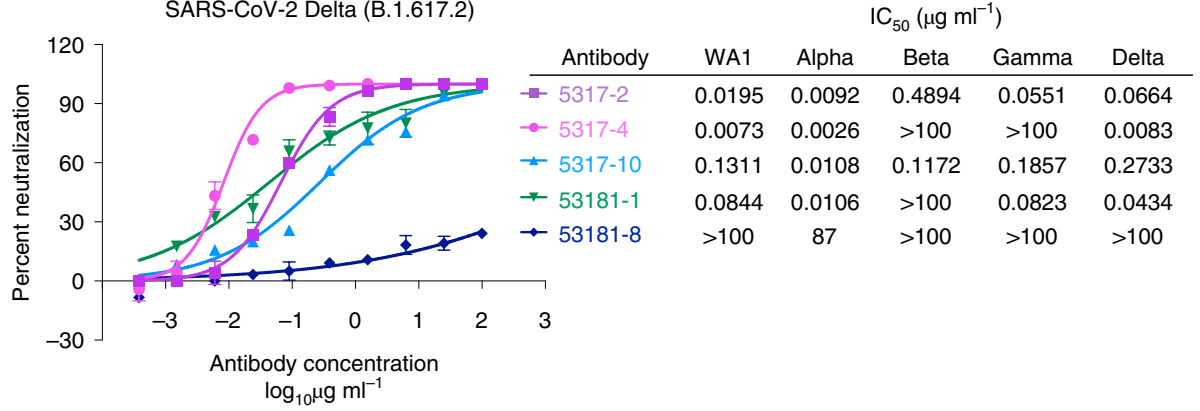

Fig. 4 | Antibody neutralization of SARS-CoV-2 variants. Authentic SARS-CoV-2 neutralization for a panel of antibodies is shown against USA-WA1 and variants (Alpha, Beta, Gamma and Delta). Data represent the percent neutralization as mean \pm s.d. The IC 50 values calculated in GraphPad Prism software by four-parameter best-fit analysis are shown on the right.

a

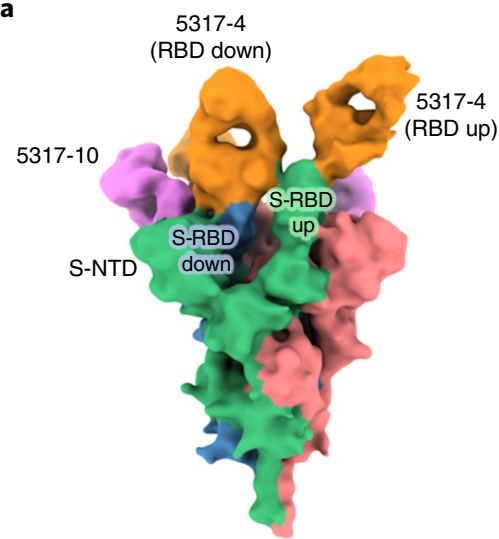

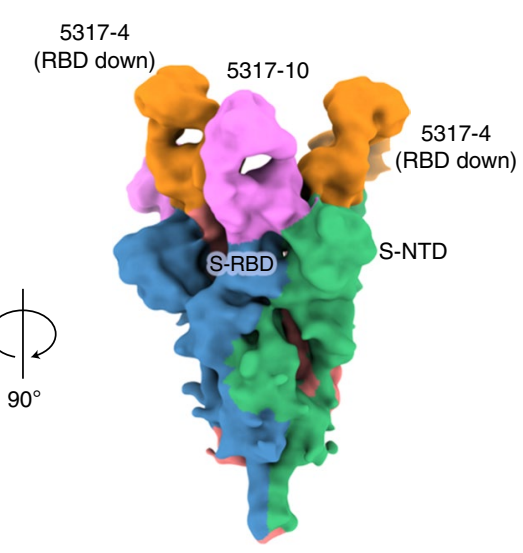

b

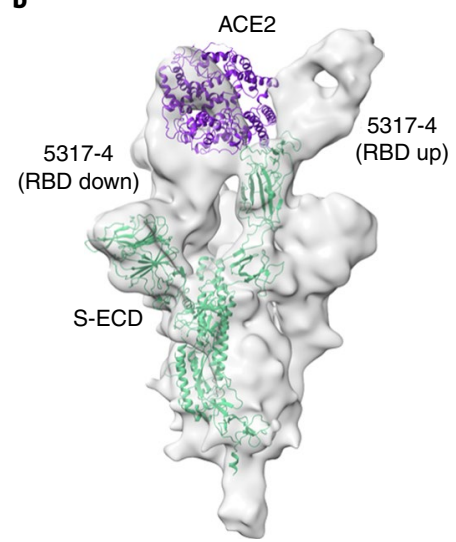

Fig. 5 | Structural characterization of antibodies 5317-4 and 5317-10. a, A 9- $\AA$-resolution cryo-EM structure of the Fab-S complex for 5317-4 Fab (orange) and 5317-10 Fab (pink). S protomers are shown in green, blue and red. b, Fab-S complex structure modeled with ACE2 (purple); ECD, extracellular domain.

The application of LIBRA-seq resulted in 828,829 and 957 antigen-specific B cells for the three experiments, respectively. We prioritized a set of $B$ cells for $\mathrm{mAb}$ production and validation based on the following conditions: for experiments 1 and 3 (with ACE2 in the screening library), we selected B cells with high LIBRA-seq scores for $\mathrm{S}$ and low scores for ACE2; for experiment 2, we selected B cells that had positive scores for multiple aliquots of S (Fig. 1b-d). B cells with high $S$ and high ACE2 scores were also selected as controls from experiments 1 and 3 , along with an influenza-specific B cell from experiment 2 (Fig. 1b-d). We further aimed to prioritize antibodies with diverse sequence features, although some of the selected antibodies appeared to be clonally related (Extended Data Fig. 2a).

We confirmed the predicted antigen specificity for 26/27 (96\%) antibodies and mapped the general antibody epitope regions by testing antibodies for binding to recombinant SARS-CoV-2 subdomain proteins (Fig. 2a and Extended Data Fig. 2b). The majority of antibodies from experiments 1 and 3 (but none from experiment 2) recognized the RBD (Fig. 2a and Extended Data Fig. $2 b$ ). Further, the antibodies had a wide range of affinities for RBD or N-terminal domain (NTD), including several antibodies with a dissociation constant $\left(K_{\mathrm{d}}\right)$ of $<1 \mathrm{nM}$, although we did not observe a correlation between LIBRA-seq S protein score and affinity (Fig. 2b). Next, we tested the ability of the antibodies to block ACE2 binding to $\mathrm{S}$ protein. For antibodies predicted to block ACE2 by LIBRA-seq, 57\% from experiment 1 and $67 \%$ from experiment 3 demonstrated ACE2 blocking via enzyme-linked immunosorbent assay (ELISA), whereas no antibodies from experiment 2 blocked ACE2 binding (Fig. 2c and Extended Data Fig. 2c).

Next, we tested the antibodies in a VSV-SARS-CoV-2 chimeric virus neutralization assay (Fig. $2 \mathrm{~d}$ and Extended Data Fig. 2d). 


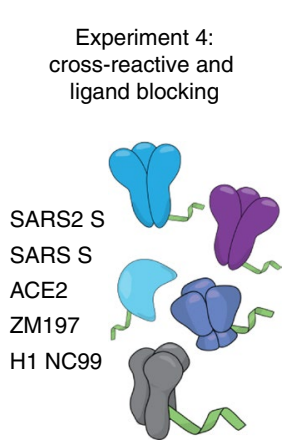

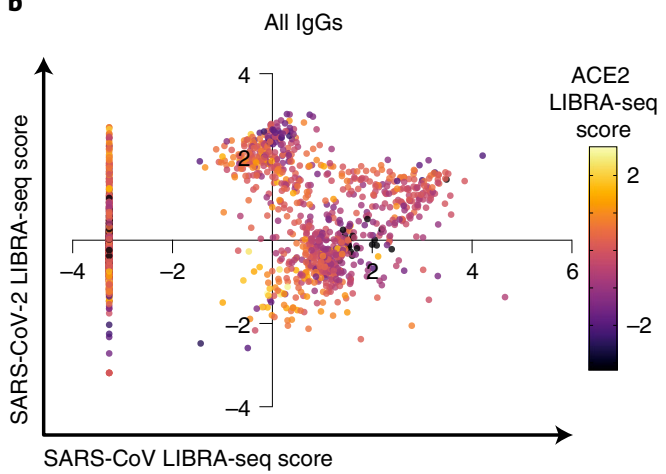

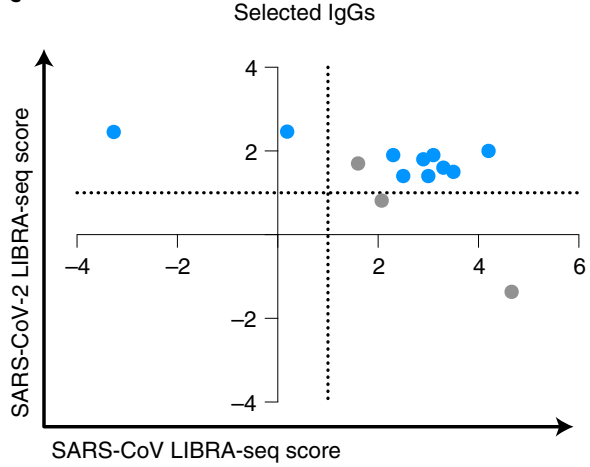

d

\begin{tabular}{|c|c|c|c|c|c|c|c|c|c|c|c|}
\hline Name & VH gene & $\mathrm{JH}$ gene & $\begin{array}{l}\text { VH } \\
\text { identity }\end{array}$ & $\begin{array}{c}\text { JH } \\
\text { identity }\end{array}$ & $\mathrm{CDRH} 3$ sequence & $\begin{array}{l}\text { CDRH3 } \\
\text { length }\end{array}$ & VL gene & JL gene & $\begin{array}{c}\text { VL } \\
\text { identity }\end{array}$ & $\underset{\text { Identity }}{\mathrm{JL}}$ & CDRL3 sequence \\
\hline $5885-1$ & IGHV4-39 & IGHJ1 & 0.96 & 0.96 & CARYTSYYDRSGFRRVEYFQHW & 20 & IGKV3-20 & IGKJ2 & 0.98 & 1.00 & CQQYGSSPYTF \\
\hline $5885-2$ & IGHV3-21 & IGHJ3 & 0.97 & 0.98 & CANMRTNYDIFTGYYPDAFDIW & 20 & IGKV3-15 & IGKJ1 & 0.98 & 1.00 & CQQYYNWPPWTF \\
\hline $5885-3$ & IGHV3-66 & IGHJ3 & 0.95 & 0.94 & CARDVTHAFDLW & 10 & IGKV1-5 & IGKJ2 & 0.97 & 1.00 & CQQYNSDLYTF \\
\hline $5885-4$ & IGHV4-38-2 & IGHJ6 & 0.97 & 0.92 & CAKEGARGRGATTSFYYYYMDVW & 21 & IGLV1-40 & IGLJ3 & 0.98 & 0.95 & CQSYDISLNGWVL \\
\hline $5885-6$ & IGHV4-61 & IGHJ6 & 0.96 & 0.82 & CARSTYYYDRSGYSTSDGMDVW & 20 & IGKV3-20 & IGKJ4 & 0.97 & 1.00 & CQQYGSSPLTF \\
\hline $5885-7$ & IGHV4-34 & IGHJ4 & 0.97 & 0.85 & CAREYSSTVWDNW & 11 & IGLV2-14 & IGLJ2 & 0.97 & 1.00 & CSSYTSSSAYVVF \\
\hline $5885-8$ & IGHV1-3 & IGHJ1 & 0.96 & 0.92 & CARPPRGYYDRTGYYNVVHYFQHW & 22 & IGKV1-33 & IGKJ4 & 0.99 & 0.97 & CQQYDNLSLTF \\
\hline $5885-9$ & IGHV1-3 & IGHJI & 0.99 & 0.90 & CARPPRGYYDRSGYYNVLLYFQHW & 22 & IGKV1-33 & IGKJ4 & 0.99 & 0.97 & CQQYVNLPLTF \\
\hline $5885-5$ & IGHVZ-30 & IGHIJ4 & 0.97 & 0.94 & CAKSEYSYAYKVHFEDYYW & 16 & IGLVE-57 & IGLJ2 & 0.98 & 0.95 & CQSYDSSNHVLF \\
\hline 5885-10 & IGHV7-4-1 & IGHJ4 & 0.95 & 0.94 & CAREDTFYFDYW & 10 & IGKV3-20 & IGKJ2 & 0.97 & 0.91 & CQQYGTSPSF \\
\hline 5885-11 & IGHV1-3 & IGHJ4 & 0.91 & 0.90 & CARGGFNYGHGLDYW & 13 & IGLV2-8 & IGLJ2 & 0.98 & 0.92 & CSSYAGVTNNLIF \\
\hline 5885-12 & IGHV3-23 & IGHJ3 & 0.98 & 0.96 & CAKYGWGLLAAAGDAFDIW & 17 & IGKV2-30 & IGKJ1 & 0.99 & 0.95 & CMQGTHWPRTF \\
\hline $5885-13$ & IGHV4-59 & IGHJ4 & 0.95 & 0.85 & CARSGSYGDRTFDHW & 13 & IGLV3-1 & IGLJ1 & 0.97 & 1.00 & CQAWGSSTAVF \\
\hline
\end{tabular}

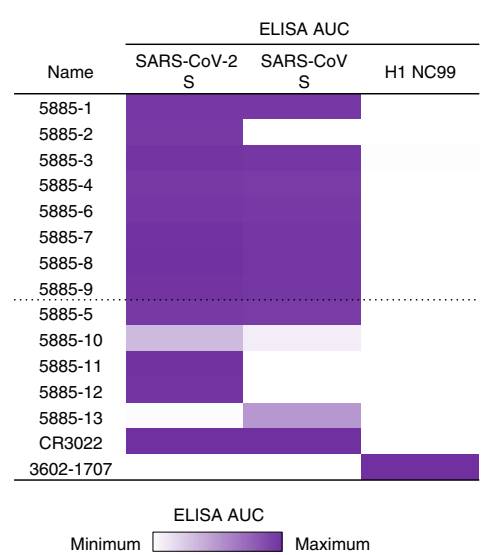

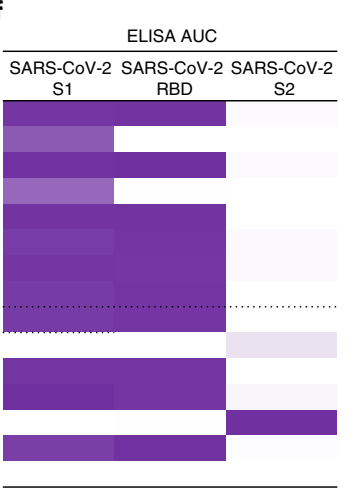

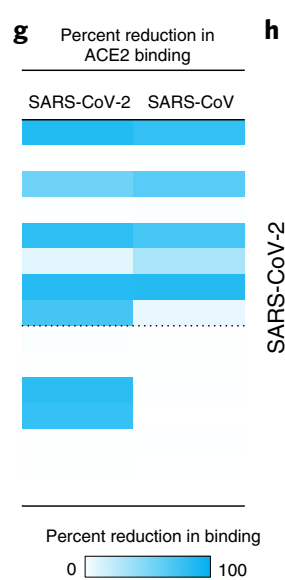

h
Percent reduction in ACE2 binding

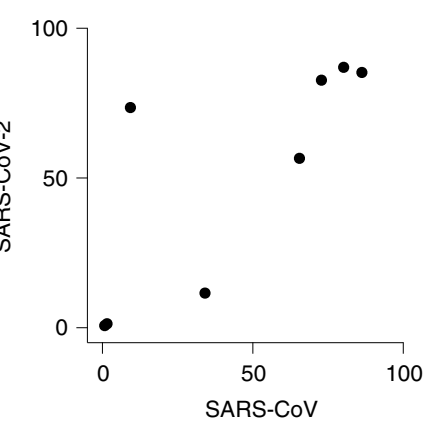

Fig. 6 | Discovery of cross-reactive ACE2-blocking coronavirus antibodies using LIBRA-seq with ligand blocking. a, Schematic of LIBRA-seq with ligand blocking applied to cross-reactive antibody discovery. $\mathbf{b}$, For identification of cross-reactive coronavirus antibodies with ligand-blocking capabilities, all IgGs recovered from the LIBRA-seq experiment $(n=2,569)$ are shown, with LIBRA-seq scores for SARS-CoV ( $x$ axis) and SARS-CoV-2 ( $y$ axis). Each dot represents a cell, and the color of the dots shows the ACE2 LIBRA-seq score, with a color heat map shown on the right. c, Cells selected for expression and validation are shown in blue (ACE2 score $<-1$ ) or gray (ACE2 score $\geq-1$ ). Of these selected cells, eight had high LIBRA-seq scores ( $>1$ ) for SARS-CoV-2 and SARS-CoV and low scores (<-1) for ACE2. Additional candidates with a variety of scores for SARS-CoV-2, SARS-CoV and ACE2 were also selected for expression and validation as controls. $\mathbf{d}$, The eight IgGs with high LIBRA-seq scores for SARS-CoV-2 and SARS-CoV and low scores for ACE2 are shown above the dotted line. Control antibodies with other LIBRA-seq score patterns are shown below the dotted line. For each antibody, complementarity-determining region (CDR) sequences and lengths are shown at the amino acid level, and $\mathrm{V}$-gene and J-gene identities are shown at the nucleotide level. LIBRA-seq scores for antigens included in the screening library (SARS-CoV-2 S, SARS-CoV S, ACE2, HIV ZM197 envelope and influenza hemagglutinin $\mathrm{H} 1 \mathrm{NC} 99$ ) are shown as a heat map; the heat map is colorized from negative (tan) to zero (white) to positive (purple). Scores outside of this range are shown as the minimum and maximum values. e,f, ELISA AUC values from binding to coronavirus $\mathrm{S}$ proteins, influenza hemagglutinin $\mathrm{H} 1$ NC99 (negative control) (e) and recombinant antigen domains (f) are shown as heat maps from minimum (white) to maximum (purple) binding. $\mathbf{g}$, Percent reduction in ACE2 binding by ELISA is shown for SARS-CoV-2 and SARS-CoV S proteins and displayed as a heat map from 0\% (white) to $100 \%$ (blue). h, For the eight IgGs with high LIBRA-seq scores for SARS-CoV-2 and SARS-CoV and low scores for ACE2, the percent reduction in ACE2 binding due to antibody blocking by ELISA is shown for SARS-CoV ( $x$ axis) and SARS-CoV-2 ( $y$ axis).

For antibodies predicted to block ACE2 by LIBRA-seq, $86 \%$ from experiment 1 and $67 \%$ from experiment 3 were neutralizing, while only two clonally related antibodies (29\%) from experiment 2 were neutralizing (Fig. 3a,b). For the antibodies from experiments 1 and 3 , the ACE2 LIBRA-seq scores were correlated with the percent reduction in ACE2 binding (Fig. 3c; Spearman $r=-0.54, P=0.017$ ). Furthermore, several antibodies also showed potent neutralization against authentic SARS-CoV-2 virus in a plaque reduction assay and in some cases against multiple SARS-CoV-2 variants (Fig. 4). Together, these results highlight the importance of including ligand 
blocking in LIBRA-seq for selectively identifying potent neutralizing antibodies.

To investigate antibody recognition of SARS-CoV-2 S, we determined a 9- $\AA$-resolution cryo-electron microscopy (cryo-EM) structure of the antigen-binding fragments of antibodies 5317-4 and 5317-10 bound to the SARS-CoV-2 S extracellular domain (Fig. 5a). We chose 5317-4 based on its potent neutralization $\left(\mathrm{IC}_{50}\right.$ value of $7.3 \mathrm{ng} \mathrm{ml}^{-1}$ against authentic SARS-CoV-2; Fig. 4) and ACE2 competition. The three-dimensional reconstruction revealed that 5317-4 binds to RBD in the 'up' and 'down' conformations, and its epitope partially overlaps the ACE2-binding footprint (Fig. 5a,b). When bound to the RBD in the down conformation, 5317-4 competes with ACE2 binding to the adjacent up RBD (Fig. 5b). We investigated 5317-10 because of its inconclusive epitope, as it bound to S1 but not individual RBD or NTD constructs (Fig. 2a). The map revealed that 5317-10 binds a quaternary epitope that bridges an RBD in the down position and the NTD of an adjacent protomer (Fig. 5a). This mode of recognition may prevent the RBD from transitioning into an ACE2-accessible up position, thereby preventing binding by ACE2.

To further demonstrate the utility of LIBRA-seq with ligand blocking, we sought to identify antibodies that show cross-reactivity between SARS-CoV-2 and SARS-CoV and that are capable of blocking S-ACE2 interactions. To that end, we applied LIBRA-seq to B cells from an individual with past SARS-CoV-2 infection by using an antigen library that included SARS-CoV-2 S, SARS-CoV S and ACE2 (Fig. 6a). This resulted in $120 \mathrm{IgG}^{+} \mathrm{B}$ cells with high LIBRA-seq scores for both SARS-CoV-2 S and SARS-CoV S (Fig. 6b). Only 8\% of these cells were associated with low LIBRA-seq scores for ACE2 (Extended Data Fig. 3a), highlighting the advantage of including ligand blocking to screen for such rare cells (although we also note that information about B cells that show cross-reactivity but are not ACE2 blocking is also retained, enabling characterization of $\mathrm{B}$ cells with alternative phenotypes as well). Based on LIBRA-seq antigen and ligand-blocking scores, we produced and validated a set of antibodies, including eight with high scores for both $\mathrm{S}$ antigens and low scores for ACE2 (Fig. 6c,d). Of these, 100\% bound SARS-CoV-2 S, $88 \%$ showed the predicted SARS-CoV-2/SARS-CoV cross-reactivity and 63\% demonstrated strong ACE2-blocking ability via ELISA (Fig. 6e-h and Extended Data Fig. 3b,c), confirming that LIBRA-seq with ligand blocking efficiently identified ACE2-blocking antibodies with cross-reactivity between multiple coronaviruses.

\section{Discussion}

Together, the results from the four LIBRA-seq experiments reported here showcase the advantages of including ligand blocking as part of the sequencing readout. As with most screening tools, there are limitations to the LIBRA-seq with ligand blocking approach, including the prerequisite for a defined antigen-ligand interaction as well as the potential for identifying false positives. Nevertheless, through a single high-throughput sequencing experiment, LIBRA-seq with ligand blocking identified potent SARS-CoV-2 antibodies, requiring the subsequent production and validation of less than a dozen antibodies per experiment. The observed hit rates for the discovery of potently neutralizing antibodies are an improvement over what has been reported in the literature, which also typically required the screening of hundreds to thousands of antibody candidates isolated for their reactivity to antigen alone (recombinant $S, S 1$ or RBD) $)^{1-6,8-12}$. Further, unlike RBD-only discovery efforts, LIBRA-seq with ligand blocking applied to $S$ antigens has the potential for more comprehensive coverage of antibody epitopes, as evidenced by the discovery of the RBD-NTD antibody in Fig. 5a. Overall, the application of LIBRA-seq with ligand blocking can provide critical advantages for rapid development of therapeutic and preventive countermeasures and presents a general platform with applications to virtually any area where targeting the disruption of antigen-ligand interaction is a prime therapeutic goal.

\section{Online content}

Any methods, additional references, Nature Research reporting summaries, source data, extended data, supplementary information, acknowledgements, peer review information; details of author contributions and competing interests; and statements of data and code availability are available at https://doi.org/10.1038/ s41587-022-01232-2.

Received: 2 June 2021; Accepted: 24 January 2022;

Published online: 3 March 2022

\section{References}

1. Jiang, S., Hillyer, C. \& Du, L. Neutralizing antibodies against SARS-CoV-2 and other human coronaviruses. Trends Immunol. 41, 355-359 (2020).

2. Zost, S. J. et al. Potently neutralizing and protective human antibodies against SARS-CoV-2. Nature 584, 443-449 (2020).

3. Chi, X. et al. A neutralizing human antibody binds to the N-terminal domain of the spike protein of SARS-CoV-2. Science 369, 650-655 (2020).

4. Brouwer, P. J. M. et al. Potent neutralizing antibodies from COVID-19 patients define multiple targets of vulnerability. Science 369, 643-650 (2020).

5. Rogers, T. F. et al. Isolation of potent SARS-CoV-2 neutralizing antibodies and protection from disease in a small animal model. Science 369, 956-963 (2020)

6. Hansen, J. et al. Studies in humanized mice and convalescent humans yield a SARS-CoV-2 antibody cocktail. Science 369, 1010-1014 (2020).

7. Krammer, F. SARS-CoV-2 vaccines in development. Nature 586, 516-527 (2020)

8. Wec, A. Z. et al. Broad neutralization of SARS-related viruses by human monoclonal antibodies. Science 369, 731-736 (2020).

9. Cohen, M. S. Monoclonal antibodies to disrupt progression of early Covid-19 infection. N. Engl. J. Med. 384, 289-291 (2021).

10. Ju, B. et al. Human neutralizing antibodies elicited by SARS-CoV-2 infection. Nature 584, 115-119 (2020).

11. Robbiani, D. F. et al. Convergent antibody responses to SARS-CoV-2 in convalescent individuals. Nature 584, 437-442 (2020).

12. Shi, R. et al. A human neutralizing antibody targets the receptor-binding site of SARS-CoV-2. Nature 584, 120-124 (2020).

13. Setliff, I. et al. High-throughput mapping of B cell receptor sequences to antigen specificity. Cell 179, 1636-1646 (2019).

Publisher's note Springer Nature remains neutral with regard to jurisdictional claims in published maps and institutional affiliations.

(C) The Author(s), under exclusive licence to Springer Nature America, Inc. 2022 


\section{Methods}

Donor information. Peripheral blood mononuclear cell (PBMC) samples were purchased from Cellero. The PBMCs were from individuals with past SARS-CoV-2 infection at least $14 \mathrm{~d}$ after symptom cessation. For experiment 1 , three samples were pooled from donors 523, 527 and 528. For experiments 2 and 3, samples from donor 523 were used for LIBRA-seq. Donor 523 had a plaque reduction neutralization test titer of 1:2,560. For experiment 4 (cross-reactive antibody discovery with ligand blocking), a sample from donor 528 was used for LIBRA-seq.

Antigen purification. A variety of recombinant soluble protein antigens were used in the LIBRA-seq experiment and other experimental assays.

Plasmids were transiently transfected in Expi293F cells using polyethylenimine and encoded the following: residues 1-1208 of the SARS-CoV-2 S protein with a mutated S1/S2 cleavage site, proline substitutions at positions $817,892,899$, 942,986 and 987 and a C-terminal T4-fibritin trimerization motif, an $8 \times$ His tag and a TwinStrepTag (SARS-CoV-2 S HexaPro (HP)); residues 1-1190 of the SARS-CoV S protein with proline substitutions at positions 968 and 969 and a C-terminal T4-fibritin trimerization motif, an $8 \times$ His tag and a TwinStrepTag (SARS-CoV S-2P) and residues 1-615 of human ACE2 with a C-terminal HRV3C protease cleavage site, a TwinStrepTag and an $8 \times$ His tag (ACE2). Transfected supernatants were collected $5 \mathrm{~d}$ after expression and purified over a StrepTactin or StrepTrap column (Cytiva Life Sciences). Both recombinant SARS-CoV-2 S HP and ACE2 were further purified to homogeneity using a Superose6 Increase column (Cytiva Life Sciences). For LIBRA-seq, non-AviTagged antigens were biotinylated using EZ-Link Sulfo-NHS-Biotin (Thermo Fisher Scientific) using a 50:1 biotin-to-protein molar ratio.

For the HIV-1 gp140 SOSIP variant from strain ZM197 (clade C) and hemagglutinin from strain A/New Caledonia/20/99 (H1N1) (GenBank accession number ACF41878), recombinant, soluble antigens contained an AviTag and were expressed in Expi $293 \mathrm{~F}$ cells using polyethylenimine transfection reagent and cultured. FreeStyle F17 expression medium supplemented with pluronic acid and glutamine was used. The cells were cultured at $37^{\circ} \mathrm{C}$ with $8 \% \mathrm{CO}_{2}$ saturation and shaking. After 5-7d, cultures were centrifuged, and the supernatant was filtered and run over an affinity column of agarose-bound Galanthus nivalis lectin. The column was washed with PBS, and antigens were eluted with $30 \mathrm{ml}$ of $1 \mathrm{M}$ methyl- $\alpha$-D-mannopyranoside. Protein elutions were buffer exchanged into PBS, concentrated and run on a Superdex 200 Increase 10/300 GL sizing column on the AKTA fast protein liquid chromatography (FPLC) system. Fractions corresponding to correctly folded protein were collected and analyzed by SDS-PAGE, and antigenicity was characterized by ELISA using known mAbs specific to each antigen. For LIBRA-seq, AviTagged antigens were biotinylated using BirA biotin ligase (Avidity).

SARS-CoV-2 S1, SARS-CoV-2 S2, SARS-CoV-2 RBD and SARS-CoV-2 NTD proteins were purchased from the commercial vendor Sino Biological.

DNA barcoding of antigens. We used oligonucleotides that possessed a 15-base pair antigen barcode, a sequence capable of annealing to the template switch oligonucleotide that is part of the 10x bead-delivered oligonucleotides and contains truncated TruSeq small RNA read 1 sequences in the following structure: 5'-CCTTGGCACCCGAGAATTCCANNNNNNNNNNNNNCCCATATAAG $A^{*} A^{*} A-3^{\prime}$, where Ns represent the antigen barcode ${ }^{13}$. For each antigen, a unique DNA barcode was directly conjugated to the antigen itself. For experiment 1 , the barcodes included SARS-CoV-2 S (GACAAGTGATCTGCA), H1 NC99 (TCATTTCCTCCGATT), ZM197 (TACGCCTATAACTTG) and ACE2 (CTTCA CTCTGTCAGG). For experiment 2, the barcodes included SARS-CoV-2 $\mathrm{S}$ aliquot 1 (GACAAGTGATCTGCA), SARS-CoV-2 $\mathrm{S}$ aliquot 2 (TGTGTATTCCCTTGT), SARS-CoV-2 $\mathrm{S}$ aliquot 3 (GCAGCGTATAAGTCA), SARS-CoV-2 $\mathrm{S}$ aliquot 4 (GCTCCTTTACACGTA), SARS-CoV-2 $\mathrm{S}$ aliquot 5 (AGACTAATAGCTGAC), SARS-CoV-2 $\mathrm{S}$ aliquot 6 (GGTAGCCCTAGAGTA), H1 NC99 (TCATTTCCTC CGATT) and ZM197 (TACGCCTATAACTTG). For experiment 3, the same barcodes were included as experiment 2 and also included ACE2 (CTTCACTCT GTCAGG). For experiment 4, the barcodes included SARS-CoV-2 S (GCAGCGT ATAAGTCA), SARS-CoV S (GCTCCTTTACACGTA), ACE2 (TACGCCTATA ACTTG), ZM197 (TCATTTCCTCCGATT) and H1 NC99 (CTTCACTCTG TCAGG). In particular, 5'-amino-oligonucleotides were conjugated directly to each antigen using the SoluLINK Protein-Oligonucleotide Conjugation kit (TriLink, S-9011) according to manufacturer's instructions. Briefly, the oligonucleotide and protein were desalted, and the amino-oligonucleotide was modified with the $4 \mathrm{FB}$ cross-linker, and the biotinylated antigen protein was modified with S-HyNic. Then, the 4FB-oligonucleotide and the HyNic-antigen were mixed. This process causes a stable bond to form between the protein and the oligonucleotide. The concentration of the antigen-oligonucleotide conjugates was determined by a bicinchoninic acid (BCA) assay, and the HyNic molar substitution ratio of the antigen-oligonucleotide conjugates was analyzed using a NanoDrop according to the SoluLINK protocol guidelines. AKTA FPLC was used to remove excess oligonucleotide from the protein-oligonucleotide conjugates, which were also verified using SDS-PAGE with a silver stain. Antigen-oligonucleotide conjugates were also used in flow cytometric titration experiments to determine optimal amounts for antigen-specific B cell sorting.
Antigen-specific B cell sorting. Cells were stained and mixed with DNA-barcoded antigens and other antibodies and sorted using FACS. First, cells were counted, and viability was assessed using trypan blue. Then, cells were washed three times with DPBS supplemented with $0.1 \%$ bovine serum albumin (BSA). Cells were resuspended in DPBS-BSA and stained with cell markers including viability dye (Ghost Red 780), CD14-APC-Cy7, CD3-FITC, CD19-BV711 and IgG-PE-Cy5. Additionally, antigen-oligonucleotide conjugates were added to the stain. For experiment 1 , oligonucleotide-labeled SARS-CoV-2 S and threefold molar excess of oligonucleotide-labeled ACE2 were added. For experiment 2, six aliquots of S protein that were each labeled with a unique DNA oligonucleotide were added in a titration series from $5 \mu \mathrm{g}$ to $0.0016 \mu \mathrm{g}$ (in fivefold dilutions). For experiment 3 , the same titration series of $S$ was added along with threefold molar excess of ACE2. For experiment 4, SARS-CoV-2 S, SARS-CoV S and threefold molar excess of oligonucleotide-labeled ACE2 were added. The antigen screening library for each of the four experiments also included an influenza virus hemagglutinin and an HIV-1 envelope variant protein as controls.

After staining in the dark for $30 \mathrm{~min}$ at room temperature, cells were washed three times with DPBS-BSA at $300 \mathrm{~g}$ for $5 \mathrm{~min}$. Cells were then incubated for $15 \mathrm{~min}$ at room temperature with Streptavidin-PE to label cells with bound antigen. Cells were washed three times with DPBS-BSA, resuspended in DPBS and sorted by FACS. Antigen-positive cells were bulk sorted and delivered to the Vanderbilt Technologies for Advanced Genomics (VANTAGE) sequencing core at an appropriate target concentration for 10x Genomics library preparation and subsequent sequencing. Flow cytometry data were analyzed using FlowJo.

\section{Sample preparation, library preparation and sequencing. Single-cell} suspensions were loaded onto the Chromium Controller microfluidics device (10x Genomics) and processed using the B cell Single Cell V(D)J solution according to the manufacturer's suggestions for a target capture of 10,000-20,000 B cells, with minor modifications to intercept, amplify and purify the antigen barcode libraries ${ }^{13}$. The 10x Genomics single-cell VDJ human B cell assay and target enrichment protocol were completed. cDNA was amplified, and additive primers were added to increase the yield of antigen-derived transcript products. After cDNA amplification, the antigen-derived transcript products were size separated from the mRNA-derived cDNA products using SPRI selection and further purification (per the manufacturer's protocol). The supernatant fraction contained the antigen-oligonucleotide-derived CDNA, whereas the beads fraction contained the full-length mRNA-derived cDNAs. After purification, the antigen-derived transcript sequencing library was prepared using a PCR reaction and purified using SPRI purification. The antigen and VDJ libraries were then analyzed, quantified and sequenced using the Illumina NovaSeq platform.

Sequence processing and bioinformatic analysis. We used our previously described pipeline to use paired-end FASTQ files of oligonucleotide libraries as input, process and annotate reads for cell barcodes, unique molecular identifiers (UMIs) and antigen barcodes and generate a cell barcode-antigen barcode UMI count matrix. B cell receptor contigs were processed using CellRanger (10x Genomics) using GRCh38 as reference. Antigen barcode libraries were also processed using CellRanger (10x Genomics). The overlapping cell barcodes between the two libraries were used as the basis of the subsequent analysis. We removed cell barcodes that had only non-functional heavy chain sequences as well as cells with multiple functional heavy chain sequences and/or multiple functional light chain sequences, reasoning that these may be multiplets. Additionally, we aligned the B cell receptor contigs (filtered_contigs.fasta file output by CellRanger, 10x Genomics) to IMGT reference genes using HighV-Quest ${ }^{14}$. The output of HighV-Quest was parsed using ChangeO ${ }^{15}$ and merged with an antigen barcode UMI count matrix. Finally, for experiments 1-3, we determined the LIBRA-seq score for each antigen in the library by calculating the centered log ratios (CLR) of each antigen UMI count for each cell. A psedocount of 1 was added to each UMI count, and the CLR was taken for each antigen for each cell. For experiment 4, the LIBRA-seq scores were calculated as previously described ${ }^{13}$. Briefly, the CLR of each antigen UMI count for each cell was calculated, and a $z$ score transformation was also performed.

Antibody expression and purification. For each antibody, variable genes were inserted into custom plasmids encoding the constant region for the IgG1 heavy chain and respective $\lambda$ - and $\kappa$-light chains (pTwist CMV BetaGlobin WPRE Neo vector, Twist Bioscience). Antibodies were expressed in Expi293F mammalian cells (Thermo Fisher Scientific) by cotransfecting heavy chain- and light chain-expressing plasmids using polyethylenimine transfection reagent and culturing for 5 to $7 \mathrm{~d}$. Cells were maintained in FreeStyle F17 expression medium supplemented at final concentrations of $0.1 \%$ Pluronic Acid F-68 and 20\% $4 \mathrm{mM}$ L-glutamine. These cells were cultured at $37^{\circ} \mathrm{C}$ with $8 \% \mathrm{CO}_{2}$ saturation and shaking. After transfection and 5-7 d of culture, cell cultures were centrifuged, and the supernatant was $0.45-\mu \mathrm{m}$ filtered with Nalgene Rapid Flow Disposable Filter Units with a PES membrane. Filtered supernatant was run over a column containing Protein A agarose resin equilibrated with PBS. The column was washed with PBS, and antibodies were eluted with $100 \mathrm{mM}$ glycine- $\mathrm{HCl}$ at $\mathrm{pH} 2.7$ directly into a 1:10 volume of $1 \mathrm{M}$ Tris- $\mathrm{HCl} \mathrm{pH} \mathrm{8.0.} \mathrm{Eluted} \mathrm{antibodies} \mathrm{were} \mathrm{buffer}$ 
exchanged into PBS three times using Amicon Ultra centrifugal filter units and concentrated. Antibody plasmids were sequenced. If antibody sequences did not match expected heavy or light chain, antibody was excluded from downstream analysis.

High-throughput antibody expression. For high-throughput production of recombinant antibodies, approaches were used that are designated as microscale. For antibody expression, microscale transfection was performed $(\sim 1 \mathrm{ml}$ per antibody) with $\mathrm{CHO}$ cell cultures using the Gibco ExpiCHO Expression System and a protocol for deep 96-well blocks (Thermo Fisher Scientific). In brief, synthesized antibody-encoding DNA ( $\sim 2 \mu \mathrm{g}$ per transfection) was added to OptiPro serum-free medium (OptiPro SFM), incubated with ExpiFectamine CHO Reagent and added to $800 \mu \mathrm{l}$ of ExpiCHO cell cultures in deep 96-well blocks using a ViaFlo384 liquid handler (Integra Biosciences). The plates were incubated on an orbital shaker at 1,000 r.p.m. with an orbital diameter of $3 \mathrm{~mm}$ at $37^{\circ} \mathrm{C}$ in $8 \% \mathrm{CO}_{2}$. The next day after transfection, ExpiFectamine CHO Enhancer and ExpiCHO Feed reagents (Thermo Fisher Scientific) were added to the cells, followed by a 4-d incubation for a total of $5 \mathrm{~d}$ at $37^{\circ} \mathrm{C}$ in $8 \% \mathrm{CO}_{2}$. Culture supernatants were collected after centrifuging the blocks at $450 \mathrm{~g}$ for $5 \mathrm{~min}$ and were stored at $4^{\circ} \mathrm{C}$ until use. For high-throughput microscale antibody purification, fritted deep-well plates were used containing $25 \mu \mathrm{l}$ of settled Protein G resin (GE Healthcare Life Sciences) per well. Clarified culture supernatants were incubated with protein $G$ resin for antibody capturing, washed with PBS using a 96-well plate manifold base (Qiagen) connected to the vacuum and eluted into 96-well PCR plates using $86 \mu \mathrm{l}$ of $0.1 \mathrm{M}$ glycine-HCl buffer, $\mathrm{pH}$ 2.7. After neutralization with $14 \mu \mathrm{l}$ of $1 \mathrm{M}$ Tris-HCl pH 8.0, purified antibodies were buffer exchanged into PBS using Zeba Spin Desalting plates (Thermo Fisher Scientific) and stored at $4{ }^{\circ} \mathrm{C}$ until use.

ELISA. To assess antibody binding, soluble protein was plated at $2 \mu \mathrm{g}$ $\mathrm{ml}^{-1}$ overnight at $4{ }^{\circ} \mathrm{C}$. The next day, plates were washed three times with PBS supplemented with $0.05 \%$ Tween- 20 (PBS-T) and coated with $5 \%$ milk powder in PBS-T. Plates were incubated for $1 \mathrm{~h}$ at room temperature and washed three times with PBS-T. Primary antibodies were diluted in $1 \%$ milk in PBS-T, starting at $10 \mu \mathrm{g}$ $\mathrm{ml}^{-1}$ with a serial 1:5 dilution and added to the plate. The plates were incubated at room temperature for $1 \mathrm{~h}$ and washed three times in PBS-T. The secondary antibody, goat anti-human IgG conjugated to peroxidase, was added at a $1: 10,000$ dilution in $1 \%$ milk in PBS-T to the plates, which were incubated for $1 \mathrm{~h}$ at room temperature. Plates were washed three times with PBS-T and developed by adding TMB substrate to each well. The plates were incubated at room temperature for $10 \mathrm{~min}$, and $1 \mathrm{~N}$ sulfuric acid was added to stop the reaction. Plates were read at $450 \mathrm{~nm}$.

Data are represented as mean \pm s.e.m. for one ELISA experiment. ELISAs were repeated two or more times. If ELISA replicates were inconsistent over more than three experiments, the antibody was excluded from in vitro characterization analysis. The AUC was calculated using Prism software version 8.0.0 (GraphPad)

ACE2 binding inhibition assay. Ninety-six-well plates were coated with $2 \mu \mathrm{g}$ $\mathrm{ml}^{-1}$ purified recombinant SARS-CoV-2 at $4{ }^{\circ} \mathrm{C}$ overnight. The next day, plates were washed three times with PBS-T and coated with $5 \%$ milk powder in PBS-T. Plates were incubated for $1 \mathrm{~h}$ at room temperature and then washed three times with PBS-T. Purified antibodies were diluted in blocking buffer at $10 \mu \mathrm{g} \mathrm{ml}^{-1}$ in triplicate, added to the wells and incubated at room temperature. Without washing, recombinant human ACE2 protein with a mouse Fc tag was added to wells for a final ACE2 concentration of $0.4 \mu \mathrm{g} \mathrm{ml}^{-1}$ and incubated for $40 \mathrm{~min}$ at room temperature. Plates were washed three times with PBS-T, and bound ACE2 was detected using horseradish peroxidase-conjugated anti-mouse Fc antibody and TMB substrate. The plates were incubated at room temperature for $10 \mathrm{~min}$, and $1 \mathrm{~N}$ sulfuric acid was added to stop the reaction. Plates were read at $450 \mathrm{~nm}$. ACE2 binding without antibody served as a control. The experiment was done in biological replicate and technical triplicates.

Biolayer interferometry. Purified antibodies were immobilized to AHC sensortips (FortéBio) to a response level of approximately $1.4 \mathrm{~nm}$ in a buffer composed of $10 \mathrm{mM}$ HEPES pH 7.5, $150 \mathrm{mM} \mathrm{NaCl}, 3 \mathrm{mM}$ EDTA, $0.05 \%$ Tween-20 and $0.1 \%$ (wt/vol) BSA. Immobilized antibodies were then dipped into wells containing twofold dilutions of either SARS-CoV-2 RBD-SD1 (residues 306-577) or SARS-CoV-2 NTD, ranging in concentration from 10 to $0.156 \mathrm{nM}$, to measure association kinetics. Dissociation kinetics were measured by dipping sensortips into wells containing only buffer. Data were reference subtracted, and kinetics were calculated in Octet Data Analysis software v.10.0 using a 1:1 binding model.

RTCA method for initial screening of antibody neutralizing activity. To screen for neutralizing activity in the panel of recombinantly expressed antibodies, we used a high-throughput and quantitative RTCA assay and a XCelligence RTCA $\mathrm{HT}$ analyzer (ACEA Biosciences) that assesses kinetic changes in cell physiology, including virus-induced cytopathic effect (CPE). Twenty microliters of cell culture medium (DMEM supplemented with $2 \%$ fetal bovine serum (FBS)) was added to each well of a 384-well E-plate using a ViaFlo384 liquid handler (Integra Biosciences) to obtain background readings. Six thousand Vero-furin cells in
$20 \mu \mathrm{l}$ of cell culture medium were seeded per well, and the plate was placed on the analyzer. Sensograms were visualized using RTCA HT software version 1.0.1 (ACEA Biosciences). For a screening neutralization assay, equal amounts of virus were mixed with microscale purified antibodies in a total volume of $40 \mu \mathrm{l}$ using DMEM supplemented with $2 \%$ FBS as a diluent and incubated for $1 \mathrm{~h}$ at $37^{\circ} \mathrm{C}$ in $5 \% \mathrm{CO}_{2}$. At $\sim 17-20 \mathrm{~h}$ after seeding the cells, the virus-antibody mixtures were added to the cells in 384-well E-plates. Wells containing virus only (in the absence of antibody) and wells containing only Vero cells in medium were included as controls. Plates were measured every $8-12 \mathrm{~h}$ for $48-72 \mathrm{~h}$ to assess virus neutralization. Microscale antibodies were assessed in four fivefold dilutions (starting from a 1:20 sample dilution), and their concentrations were not normalized. Neutralization was calculated as the percentage of maximal cell index in control wells without virus minus cell index in control (virus-only) wells that exhibited maximal CPE at $40-48 \mathrm{~h}$ after applying virus-antibody mixture to the cells. An antibody was classified as fully neutralizing if it completely inhibited SARS-CoV-2-induced CPE at the highest tested concentration, while an antibody was classified as partially neutralizing if it delayed but did not fully prevent CPE at the highest tested concentration ${ }^{2,16}$.

RTCA neutralization assay. To determine neutralizing activity of IgG, we used an RTCA assay on an xCELLigence RTCA MP analyzer (ACEA Biosciences) that measures virus-induced CPE ${ }^{17}$. Briefly, $50 \mu \mathrm{l}$ of cell culture medium (DMEM supplemented with $2 \%$ FBS) was added to each well of a 96 -well E-plate using a ViaFlo384 liquid handler (Integra Biosciences) to obtain background readings. A suspension of 18,000 Vero E6 cells in $50 \mu \mathrm{l}$ of cell culture medium was seeded in each well, and the plate was placed on the analyzer. Measurements were taken automatically every $15 \mathrm{~min}$, and the sensograms were visualized using RTCA software version 2.1.0 (ACEA Biosciences). VSV-SARS-CoV-2 (0.01 multiplicity of infection, $\sim 120$ plaque-forming units (p.f.u.) per well) was mixed 1:1 with a dilution of antibody in a total volume of $100 \mu \mathrm{l}$ using DMEM supplemented with $2 \% \mathrm{FBS}$ as a diluent and incubated for $1 \mathrm{~h}$ at $37^{\circ} \mathrm{C}$ in $5 \% \mathrm{CO}_{2}$. At $16 \mathrm{~h}$ after seeding the cells, the virus-antibody mixtures were added in replicates to the cells in 96-well E-plates. Triplicate wells containing virus only (maximal CPE in the absence of antibody) and wells containing only Vero cells in medium (no-CPE wells) were included as controls. Plates were measured continuously (every $15 \mathrm{~min}$ ) for $48 \mathrm{~h}$ to assess virus neutralization. Normalized cellular index values at the endpoint ( $48 \mathrm{~h}$ after incubation with the virus) were determined using the RTCA software version 2.1.0 (ACEA Biosciences). Results are expressed as percent neutralization in a presence of respective antibody relative to control wells with no CPE minus cellular index values from control wells with maximum CPE. RTCA $\mathrm{IC}_{50}$ values were determined by non-linear regression analysis using Prism software.

Plaque reduction neutralization test. Virus neutralization with live authentic SARS-CoV-2 virus (USA-WA1) was performed in the BSL-3 facility of the Galveston National Laboratory using Vero E6 cells (ATCC, CRL-1586) following the standard procedure. Vero E6 cells were cultured in 96-well plates $\left(10^{4}\right.$ cells per well). The next day, fourfold serial dilutions of antibodies were made using MEM supplemented with $2 \%$ FBS to get an initial concentration of $100 \mu \mathrm{g} \mathrm{ml}^{-1}$. Equal volumes of diluted antibodies $(60 \mu \mathrm{l})$ were mixed gently with original SARS-CoV-2 (USA-WA1) $\left(60 \mu \mathrm{l}\right.$ containing 200 p.f.u.) and incubated for $1 \mathrm{~h}$ at $37^{\circ} \mathrm{C}$ and $5 \%$ $\mathrm{CO}_{2}$ atmosphere. The virus-serum mixture $(100 \mu \mathrm{l})$ was added to cell monolayers in duplicates and incubated for $1 \mathrm{~h}$ at $37^{\circ} \mathrm{C}$ and $5 \% \mathrm{CO}_{2}$ atmosphere. Later, the virus-serum mixture was discarded gently, and the cell monolayer was overlaid with $0.6 \%$ methylcellulose and incubated for $2 \mathrm{~d}$. The overlay was removed, and the plates were fixed in $4 \%$ paraformaldehyde twice following BSL-3 protocol. The plates were stained with $1 \%$ crystal violet, and virus-induced plaques were counted. The percent neutralization and/or neutralizing antibody titer at 50\% inhibition $\left(\mathrm{NT}_{50}\right)$ of antibody was calculated by dividing the plaques counted at each dilution with plaques of virus-only control. For antibodies, the $\mathrm{IC}_{50}$ values were calculated in Prism software (GraphPad) by plotting the midway point between the upper and lower plateaus of the neutralization curve among dilutions. The Alpha variant virus incorporates the following substitutions: deletion 69-70, deletion 144, E484K, N501Y, A570D, D614G, P681H, T716I, S982A and D1118H. The Beta variant incorporates the following substitutions: deletion 24, deletion 242-243, D80A, D215G, K417N, E484K, N501Y, D614G, H665Y and T1027I. The Gamma variant incorporates the following substitutions: L18F, T20N, P26S, D138Y, R190S, K417T, E484K, N501Y, D614G, H655Y and T1027I. The Delta variant incorporates the following substitutions: T19R, G142D, deletion 156-157, R158G, L452R, T478K, D614G, P681R, deletion 689-691 and D950N; the deletion at positions 689-691 has not been observed in nature, and was identified following one passage of the virus.

Fab preparation. To generate Fabs, IgGs were incubated with Lys-C at 1:4,000 (wt/ wt) overnight at $37^{\circ} \mathrm{C}$. EDTA-free protease inhibitor (Roche) was dissolved to $25 \times$ and added to the sample at a final $1 \times$ concentration. The sample was passed over a Protein A column. The flow-through was collected and run on a Superdex 200 Increase 10/300 GL sizing column on the AKTA FPLC system. Fabs were visualized by SDS-PAGE. 
Electron microscopy sample preparation and data collection. Purified SARS-CoV-2 S HP ectodomain ${ }^{18}$ and Fabs 5317-4 and 5317-10 were combined at a final complex concentration of $0.4 \mathrm{mg} \mathrm{ml}^{-1}$. Fab 5317-10 was added to $\mathrm{S}$ and incubated on ice for 30 min before the addition of Fab 5317-4 immediately before grid deposition and freezing. The complex was deposited on Au-300 1.2/1.3 grids that had been plasma cleaned for $4 \mathrm{~min}$ in a Solarus 950 plasma cleaner (Gatan) with a 4:1 ratio of $\mathrm{O}_{2} / \mathrm{H}_{2}$. Excess liquid was blotted for $3 \mathrm{~s}$ with a force of -4 using a Vitrobot Mark IV (Thermo Fisher) and plunge-frozen into liquid ethane. A total of 2,655 micrographs were collected from a single grid with the stage at a $30^{\circ}$ tilt using a Titan Krios (Thermo Fisher) equipped with a K3 detector (Gatan). Movies were collected using SerialEM ${ }^{19}$ at $\times 29,000$ magnification with a corresponding calibrated pixel size of $0.81 \AA$ per pixel.

Cryo-EM. Motion correction, contrast transfer function estimation, particle picking and two-dimensional classification were performed using cryoSPARC v.3.2.0 $\left(\right.$ ref. $\left.{ }^{20}\right)$. The final iteration of two-dimensional class averaging distributed 17,710 particles into 50 classes using an uncertainty factor of 3 . From that, 13,232 particles were selected, and an ab inito reconstruction was performed with four classes followed by heterogeneous refinement of those four classes. A total of 6,803 particles from the highest-quality class were used for homogenous refinement of the best volume without imposed symmetry. The resulting volume was used for an additional round of homogenous refinement. To filter out additional junk particles, an ab initio reconstruction was performed with three classes followed by heterogeneous refinement of those three classes. A total of 5,171 particles from the highest-quality class were used for homogenous refinement of the best volume without imposed symmetry, resulting in a final 9-Å map.

Quantification and statistical analysis. ELISA error bars (s.e.m.) were calculated using GraphPad Prism version 8.0.0. Spearman $r$ correlation was performed using GraphPad Prism 8.0.0. Analysis of variance was performed for neutralization potency comparisons using GraphPad Prism version 8.0.0.

Reporting Summary. Further information on research design is available in the Nature Research Reporting Summary linked to this article.

\section{Data availability}

All unique reagents generated in this study are available from the corresponding author with a completed material transfer agreement (a template can be found in the manuscript files). Sequences for antibodies identified and characterized in this study have been deposited to GenBank (MZ517191-MZ517250, OM001674OM001699). Raw sequencing data has been deposited to Sequence Read Archive (PRJNA744567, SAMN24369247). Cryo-EM maps for the 3D reconstruction of trimeric SARS-CoV-2 S bound to Fabs 5713-4 and 5713-10 have been deposited with the Electron Microscopy Data Bank under accession code EMD-26064. Further information and requests for resources and reagents should be directed to the corresponding author.

\section{Code availability}

Custom scripts used to analyze data in this manuscript are available at https:// github.com/Iglab-repo/LIBRA-seq-with-ligand-blocking.git. An end user license agreement can be found in the manuscript files.

\section{References}

14. Alamyar, E., Duroux, P., Lefranc, M. P. \& Giudicelli, V. IMGT tools for the nucleotide analysis of immunoglobulin (IG) and T cell receptor (TR) V-(D)-J repertoires, polymorphisms, and IG mutations: IMGT/V-QUEST and IMGT/ HighV-QUEST for NGS. Methods Mol. Biol. 882, 569-604 (2012).

15. Gupta, N. T. et al. Change-O: a toolkit for analyzing large-scale B cell immunoglobulin repertoire sequencing data. Bioinformatics 31, 3356-3358 (2015).

16. Gilchuk, P. et al. Integrated pipeline for the accelerated discovery of antiviral antibody therapeutics. Nat. Biomed. Eng. 4, 1030-1043 (2020).

17. Suryadevara, N. et al. Neutralizing and protective human monoclonal antibodies recognizing the N-terminal domain of the SARS-CoV-2 spike protein. Cell 184, 2316-2331 (2021).
18. Hsieh, C. L. et al. Structure-based design of prefusion-stabilized SARS-CoV-2 spikes. Science 369, 1501-1505 (2020).

19. Mastronarde, D. N. Automated electron microscope tomography using robust prediction of specimen movements. J. Struct. Biol. 152, 36-51 (2005).

20. Punjani, A., Rubinstein, J. L., Fleet, D. J. \& Brubaker, M. A. cryoSPARC: algorithms for rapid unsupervised cryo-EM structure determination. Nat. Methods 14, 290-296 (2017).

\section{Acknowledgements}

We thank A. Jones, L. Raju and J. Roberson of VANTAGE for their expertise regarding next-generation sequencing and library preparation, D. Flaherty and B. Matlock of the Vanderbilt Flow Cytometry Shared Resource for help with flow panel optimization and members of the Georgiev laboratory for comments on the manuscript. The Vanderbilt VANTAGE Core provided technical assistance for this work. VANTAGE is supported, in part, by CTSA grant 5UL1 RR024975-03, the Vanderbilt Ingram Cancer Center (P30 CA68485), the Vanderbilt Vision Center (P30 EY08126) and NIH/NCRR (G20 RR030956). This work was conducted, in part, using the resources of the Advanced Computing Center for Research and Education at Vanderbilt University. Flow cytometry experiments were performed in the Vanderbilt University Medical Center (VUMC) Flow Cytometry Shared Resource. The VUMC Flow Cytometry Shared Resource is supported by the Vanderbilt Ingram Cancer Center (P30 CA68485) and the Vanderbilt Digestive Disease Research Center (DK058404). For work described in this manuscript, I.S.G., A.R.S., K.J.K., S.C.W., K.A.P., N.R. and L.M.W. were supported, in part, by NIH National Institute of Allergy and Infectious Diseases (NIAID) award R01AI131722-S1, the Hays Foundation COVID-19 Research Fund, Fast Grants and CTSA award number UL1 TR002243 from the National Center for Advancing Translational Sciences. J.S.M, N.V.J. and D.W. were supported, in part, by an NIH NIAID grant awarded to J.S.M. (R01-AI127521), a Welch Foundation grant F-0003-19620604 and an NIH NIAID award R01AI131722-S1. S.P. and A.B. were supported, in part, by the NIAID grants U19AI142785 and U19 AI142790. J.E.C., N.S., R.N., R.E.S. and R.H.C. were supported, in part, by Defense Advanced Research Projects Agency (DARPA) grant HR0011-18-20001, US NIH contract 75N93019C00074, NIH grant R01 AI157155, the Dolly Parton COVID-19 Research Fund at Vanderbilt and a grant from Fast Grants, Mercatus Center, George Mason University. J.E.C. is a recipient of the 2019 Future Insight Prize from Merck KGaA, which supported this work with a grant.

\section{Author contributions}

A.R.S., I.S. and I.S.G. developed the methodology. A.R.S., K.J.K., N.V.J., S.C.W., N.S., D.W., S.P., K.A.P., N.R., R.N., R.E.S., L.M.W., J.E.C., A.B., R.H.C., J.S.M. and I.S.G. performed the investigations. A.R.S. and N.R. developed software. A.R.S. and K.J.K. performed validations. A.R.S. and I.S.G. wrote the original draft. All authors reviewed and edited the manuscript. I.S.G., J.E.C., A.B., R.H.C., J.S.M., A.R.S. and K.J.K. acquired funding. I.S.G., J.E.C., A.B., R.H.C. and J.S.M. provided resources. I.S.G. supervised the work.

\section{Competing interests}

A.R.S. and I.S.G. are cofounders of AbSeek Bio. I.S.G., A.R.S. and K.J.K. are listed as inventors on antibodies described herein. I.S.G., A.R.S. and I.S. are listed as inventors on patent applications for the LIBRA-seq technology. J.E.C. has served as a consultant for Luna Biologics, is a member of the Scientific Advisory Board Meissa Vaccines and is Founder of IDBiologics. The Crowe laboratory has received funding support in sponsored research agreements from AstraZeneca, IDBiologics and Takeda. The Georgiev laboratory at VUMC has received unrelated funding from Takeda Pharmaceuticals. The remaining authors declare no competing interests.

\section{Additional information}

Extended data is available for this paper at https://doi.org/10.1038/s41587-022-01232-2.

Supplementary information The online version contains supplementary material available at https://doi.org/10.1038/s41587-022-01232-2.

Correspondence and requests for materials should be addressed to Ivelin S. Georgiev.

Peer review information Nature Biotechnology thanks Laura Walker, Daniel Lingwood and the other, anonymous, reviewer(s) for their contribution to the peer review of this work.

Reprints and permissions information is available at www.nature.com/reprints. 


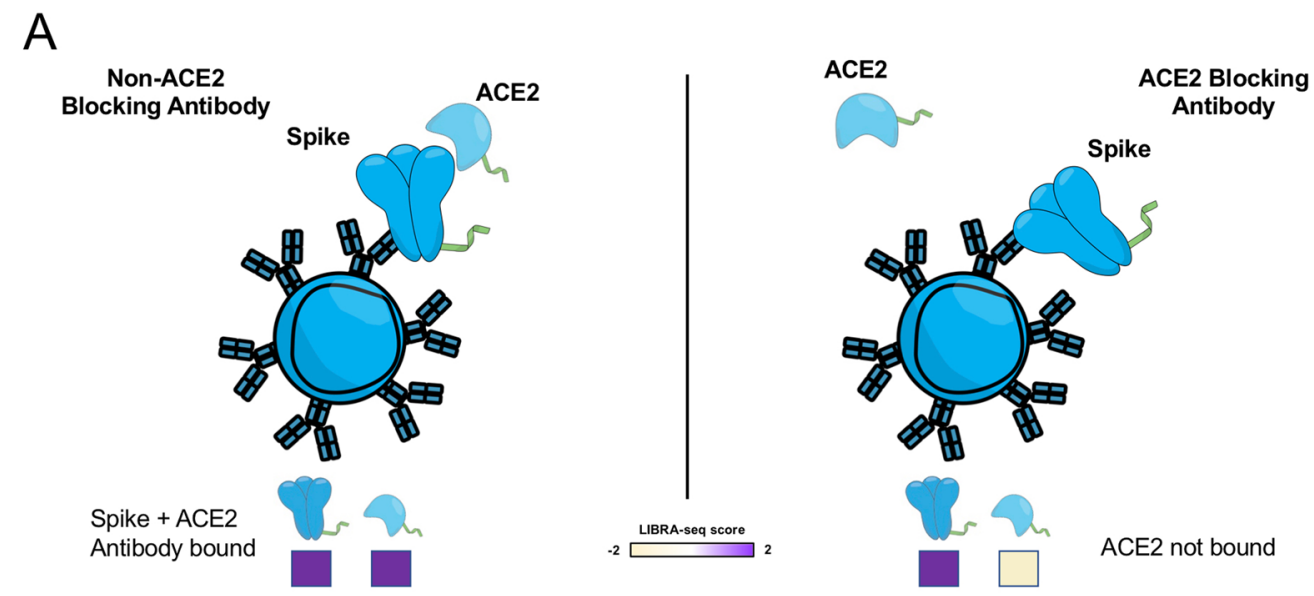

\section{B}

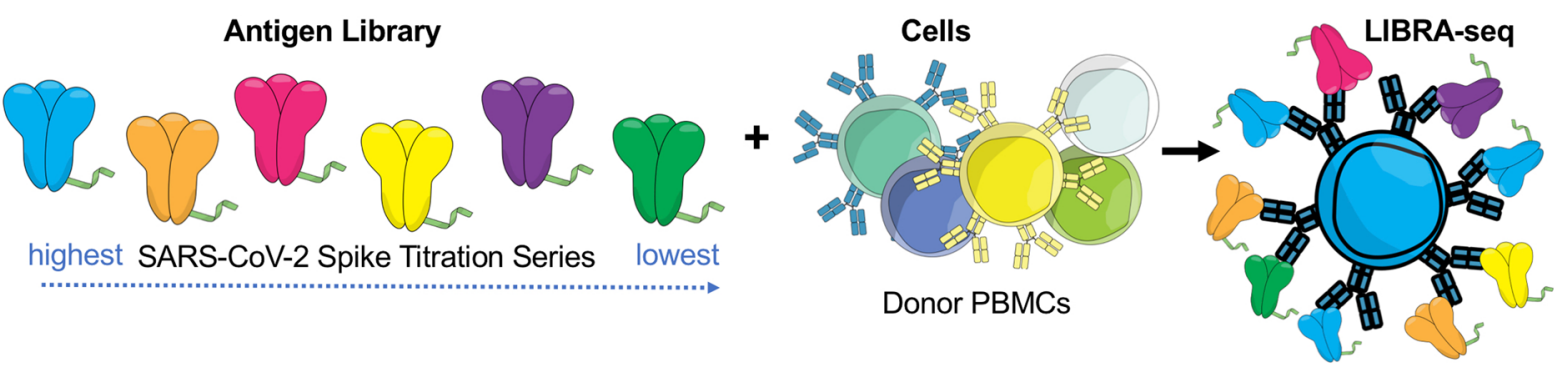

highest SARS-CoV-2 Spike Titration Series lowest

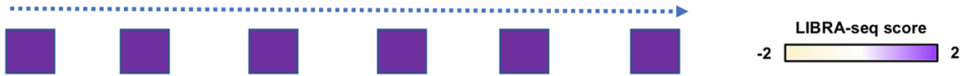

C

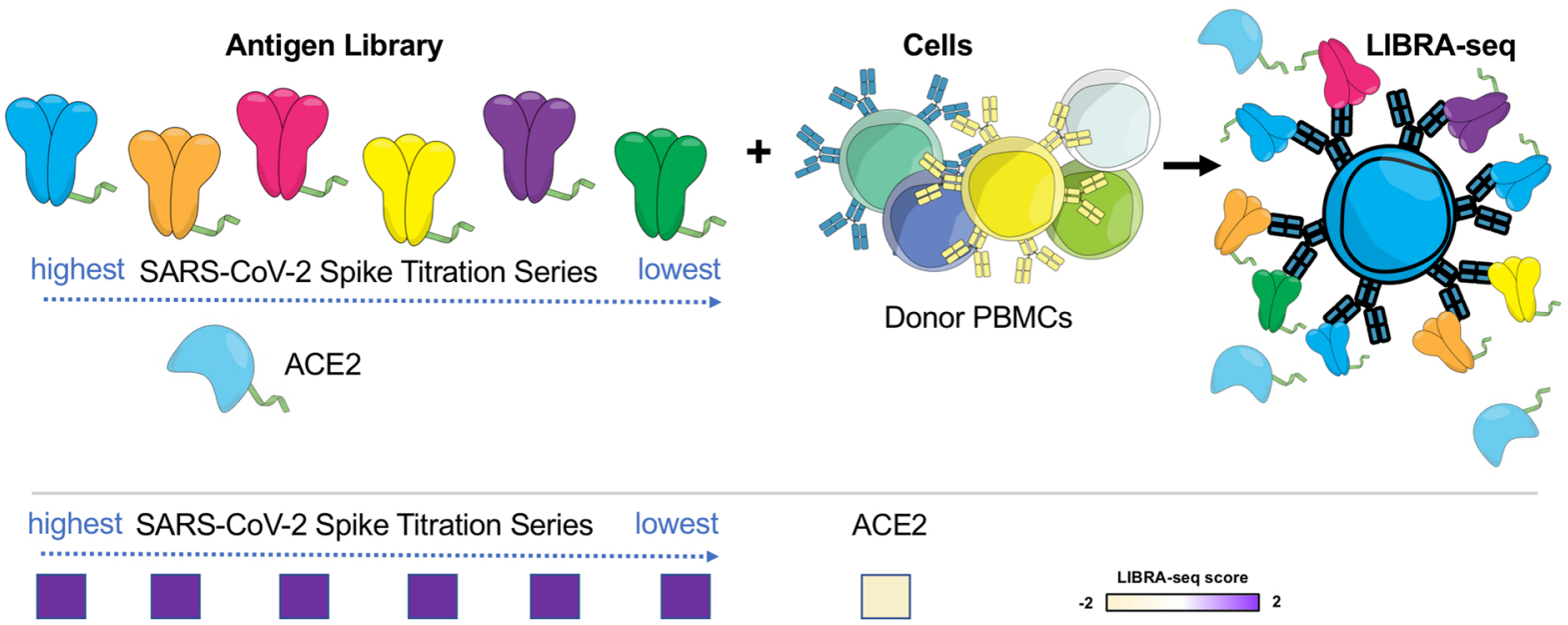

Extended Data Fig. 1 | Schematic representation of LIBRA-seq experiments. a. An antigen screening library of oligonucleotide-labeled antigens was generated. This library consisted of SARS-CoV-2 spike antigens and negative controls. Additionally, oligo-labeled ACE2 (the SARS-CoV-2 spike host cell receptor) was included. The antigen screening library was mixed with donor PBMCs. This approach allowed for assessment of B cell ligand blocking functionality from the sequencing experiment. $\mathbf{b}$. An antigen screening library containing an antigen titration was generated, with a goal of identifying high affinity antibodies from LIBRA-seq. In this experiment, six different amounts of oligo-labeled SARS-CoV-2 S protein, each labeled with a different barcode, were included in a screening library. C. Schematic of LIBRA-seq with S titrations and ACE2 included for ligand blocking. 
A

\begin{tabular}{|c|c|c|c|c|c|c|c|c|c|c|c|c|}
\hline Antibody & $\mathrm{VH}$ Gene & JH Gene & VH Identity & JH Identity & CDRH3 Sequence & $\begin{array}{l}\text { CDRH3 } \\
\text { Length }\end{array}$ & VL Gene & JL Gene & VL Identity & JL Identity & y CDRL3 Sequence & $\begin{array}{l}\text { CDRL3 } \\
\text { Length }\end{array}$ \\
\hline $5317-1$ & IGHV1-58 & IGHJ4 & 0.95 & 0.88 & CAADPFADYW & 8 & IGLV1-44 & IGLJ3 & 0.98 & 0.95 & CATWDDSLNAWVF & 11 \\
\hline $5317-2$ & IGHV1-69 & IGHJ5 & 0.95 & 0.92 & CARGLWFGDSETVWFDPW & 16 & IGKV1-39 & IGKJ1 & 0.97 & 0.97 & CQQSYSTPPTF & 9 \\
\hline $5317-3$ & IGHV3-64D & IGHJ4 & 0.93 & 0.88 & CVKGKIQLWLGADYW & 13 & IGKV1-39 & IGKJ1 & 0.97 & 1.00 & CQQSYNTPWTF & 9 \\
\hline $5317-4$ & IGHV4-34 & IGHJ3 & 0.93 & 0.88 & CARKPLLHSSVNPGAFDIW & 17 & IGKV3-20 & IGKJ2 & 0.96 & 0.92 & CQQYATSPRTF & 9 \\
\hline $5317-5$ & IGHV1-69 & IGHJ4 & 0.97 & 0.96 & CAREKGYSSSSSATYYLDFW & 18 & IGLV1-40 & IGLJ2 & 0.98 & 0.95 & CQSYDSSLTALVF & 11 \\
\hline $5317-6$ & IGHV4-39 & IGHJ6 & 0.97 & 0.84 & CARRVPGDYYCLDVW & 13 & IGKV1-39 & IGKJ4 & 0.96 & 0.95 & CQQSFSARVPTF & 10 \\
\hline $5317-7$ & IGHV3-7 & IGHJ4 & 0.97 & 0.88 & CARGGLWGTFDYW & 11 & IGKV3-20 & IGKJ2 & 0.98 & 1.00 & CQQFAYSLYTF & 9 \\
\hline $5317-8$ & IGHV3-30-3 & IGHJ6 & 0.96 & 0.87 & CARAYGGNYYYGMDVW & 14 & IGLV3-1 & IGLJ1 & 0.96 & 0.97 & CQAWDSSTASFVF & 11 \\
\hline $5317-9$ & IGHV1-69 & IGHJ5 & 0.95 & 0.84 & CASLGGDSYISGTHYDRSGYDPW & 21 & IGKV3-11 & IGKJ4 & 0.98 & 0.95 & CQRRSNWPPFTF & 10 \\
\hline$\underline{5317-10}$ & IGHV5-10-1 & IGHJ4 & 0.93 & 0.81 & CARVNRVGDGPDFW & 12 & IGKV2-28 & IGKJ1 & 0.97 & 1.00 & CMQALQTPWTF & 9 \\
\hline $53181-1$ & IGHV1-18 & IGHJ6 & 0.97 & 0.95 & CARDPASYYDFWSGYVDYYYYGMDVW & 24 & IGKV3-20 & IGKJ3 & 0.99 & 1.00 & CQQYGNSRLTF & 9 \\
\hline $53181-2$ & IGHV1-18 & IGHJ6 & 0.99 & 0.95 & CARDPASYYDLWSGYVDYYYYGMDVW & 24 & IGKV3-20 & IGKJ3 & 0.99 & 1.00 & CHHYGSSRLTF & 9 \\
\hline $53181-3$ & IGHV1-69 & IGHJ4 & 0.91 & 0.75 & CARSGGYRLWFGELW & 13 & IGKV3-20 & IGKJ5 & 0.94 & 0.95 & CQQYGGSPATF & 9 \\
\hline $53181-4$ & IGHV3-33 & IGHJ4 & 0.98 & 0.88 & CAREGAVGATSGLDYW & 14 & IGLV3-10 & IGLJ3 & 0.99 & 0.89 & CYSRDSSGNPLF & 10 \\
\hline $53181-5$ & IGHV4-59 & IGHJ4 & 0.95 & 0.96 & CARGFDYW & 6 & IGKV3-20 & IGKJ1 & 0.98 & 1.00 & CQQYGSSPWTF & 9 \\
\hline $53181-6$ & IGHV4-59 & IGHJ6 & 0.94 & 0.89 & CARGAGEQRLVGGLFGVSHFYYYMDVW & 25 & IGKV1-5 & IGKJ1 & 0.99 & 1.00 & CQQYNSYPWTF & 9 \\
\hline $53181-7$ & IGHV3-23 & IGHJ4 & 0.94 & 0.81 & CAKSATIVLMVSAIYW & 14 & IGLV2-14 & IGLJ2 & 0.97 & 1.00 & CSSYTSTSTLVF & 10 \\
\hline $53181-8$ & IGHV3-72 & IGHJ6 & 0.99 & 0.94 & CARVRGGEWVGDLGWYYYYGMDWW & 22 & IGKV2-28 & IGKJ2 & 0.99 & 0.97 & CMQALQTPRTF & 9 \\
\hline$\underline{53181-9}$ & IGHV3-30 & IGHJ4 & 0.91 & 0.92 & CVKGATKIDYW & 9 & IGLV2-14 & IGLJ1 & 0.91 & 0.83 & CFSYTSGGTRVF & 10 \\
\hline $53182-1$ & IGHV3-53 & IGHJ3 & 0.95 & 0.96 & CTRGGWPSGDTFDIW & 13 & IGKV1-9 & IGKJ3 & 0.99 & 0.97 & CQQLNSYPEITF & 10 \\
\hline $53182-3$ & IGHV3-66 & IGHJ6 & 0.99 & 0.87 & CARDRRIIGYYFGMDVW & 15 & IGLV2-23 & IGLJ3 & 0.97 & 1.00 & CCPYADTWVF & 8 \\
\hline $53182-4$ & IGHV2-5 & $I G H J 3$ & 0.85 & 0.86 & CARLLIEHDAFDIW & 12 & IGKV2-28 & IGKJ2 & 0.92 & 0.97 & CMQALHFPYTF & 9 \\
\hline $53182-5$ & IGHV1-69 & IGHJ4 & 0.85 & 0.88 & CAREEGSGWWKHDYW & 13 & IGKV1-9 & IGKJ2 & 0.97 & 1.00 & CQQLSGYPYTF & 9 \\
\hline $53182-6$ & IGHV3-66 & IGHJ6 & 0.95 & 0.84 & CVRDRRIVGYYFGLDVW & 15 & IGLV2-23 & IGLJ3 & 0.96 & 0.95 & CCSYATTWVF & 8 \\
\hline $53182-7$ & IGHV3-23 & IGHJ6 & 0.98 & 0.97 & CAKDAFYYGSGSHFYYYYYMDVW & 21 & IGKV3-20 & IGKJ5 & 0.99 & 0.97 & CQQYGSSPTF & 8 \\
\hline $53182-8$ & IGHV4-39 & IGHJ4 & 0.99 & 0.92 & CARDRRGGGWTASFDFW & 15 & IGKV4-1 & IGKJ2 & 0.99 & 0.97 & CQQHYSTPGYTF & 10 \\
\hline 53182-9 & IGHV3-53 & IGHJ3 & 0.98 & 0.98 & CARGGWPSGDTFDIW & 13 & IGKV1-9 & IGKJ3 & 0.99 & 0.97 & CQQLNSYPEITF & 10 \\
\hline $53182-10$ & IGHV2-5 & IGHJ4 & 0.98 & 0.83 & CAHHTVPTIYDYW & 11 & IGLV2-8 & IGLJ3 & 0.98 & 0.97 & CSSYAGSNPLVF & 10 \\
\hline $53182-11$ & IGHV3-9 & IGHJ3 & 0.93 & 0.92 & CAKDIGRYDHYNIFGRVGGAFDIW & 22 & IGKV1-33 & IGKJ3 & 0.95 & 0.94 & CQHYDNLPRF & 8 \\
\hline
\end{tabular}

B
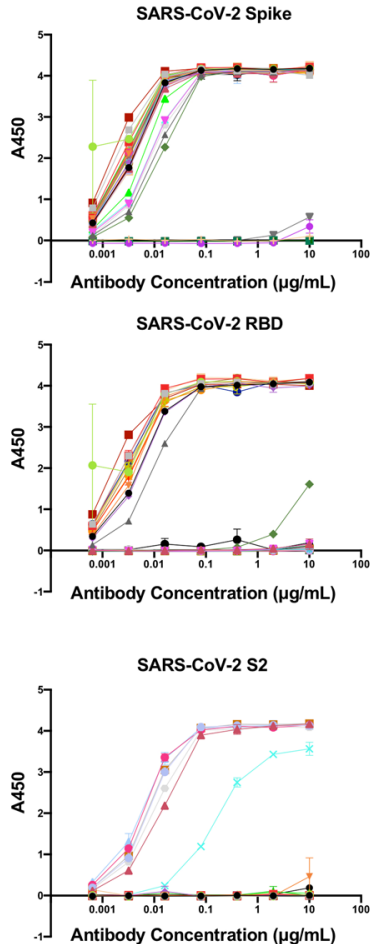

$\bullet 5317-1$

- $5317-2$

$-5317-3$

$-5317-4$

$\leftarrow$ 5317-5

- $5317-6$

- $5317-7$

- $5317-8$

- 5317-9

- 5317-10

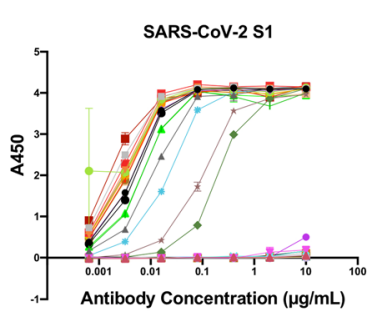

SARS-CoV-2 NTD
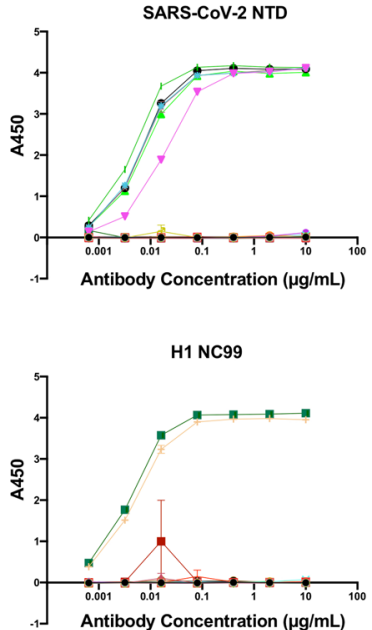

$\begin{array}{ll}-53182-1 & - \text { CR3022 } \\ +53182-3 & - \text { NTD mAb } \\ +53182-4 & - \text { S2 mAb } \\ -53182-5 & -3602-1707 \\ -53182-6 & -1 \text { - } 1 \text { F } \\ -53182-7 & \\ -53182-8 & \\ -53182-9 & \\ -53182-11 & \end{array}$

C

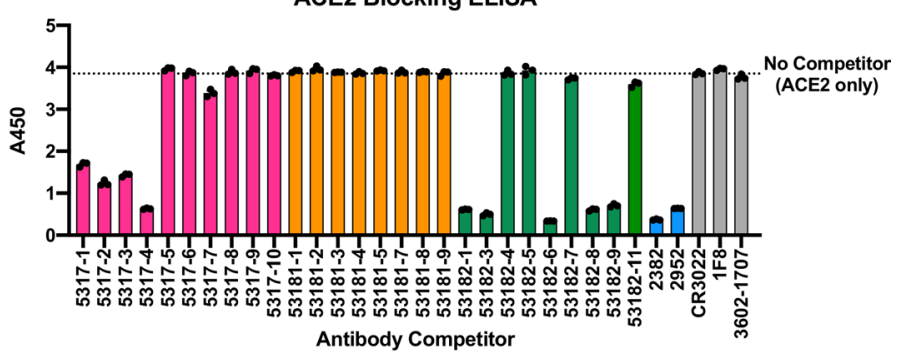

D

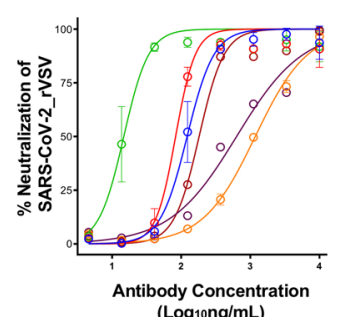

(Log10ng/mL)

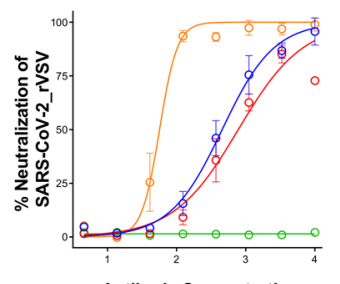

Antibody Concentration (Log10ng/mL)

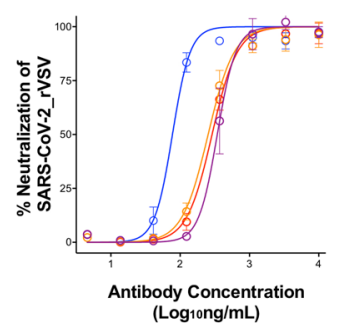

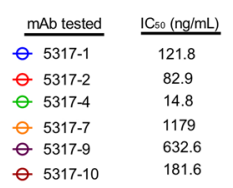

mAb tested $\quad$ IC $\mathrm{Cs}_{0}(\mathrm{no} / \mathrm{mL})$

$\theta$ 53181-1 456

$\theta 53181-2 \quad 780$

r. Positive control 50

Extended Data Fig. 2 | See next page for caption. 
Extended Data Fig. 2 | Characterization of LIBRA-seq-identified antibodies. a. Genetic characteristics for monoclonal antibodies prioritized for expression and validation. $V_{H}, J_{H}, V_{L}, J_{L}$ inferred gene segment identity is shown at the nucleotide level. CDRH3 and CDRL3 amino acid sequence and length are also shown. b. ELISA binding of antibodies to SARS-CoV-2 spike, SARS-CoV-2 S1, SARS-CoV-2 RBD, SARS-CoV-2 NTD, SARS-CoV-2 S2 and influenza hemagglutinin H1 NC99. Data are represented as mean \pm SEM of technical duplicates and represent one of at least two independent experiments $(n=2)$. c. ACE2 blocking ELISA. Antibodies were added to spike, and recombinant ACE2 was added and detected. Antibodies that block ACE2 binding show a reduction in absorbance compared to ACE2 binding without competitor (dotted line). ELISAs were performed at one antibody concentration, and data are represented as mean \pm SEM of technical triplicates and represent one of at least two independent experiments $(n=2)$. $d$. Antibodies were tested in a VSV SARS-CoV-2 real time cell analysis (RTCA) neutralization assay. Neutralization curves and IC50 values are shown. Data are represented as mean \pm S.D. of technical triplicates, and represent one of two independent experiments $(n=2)$. 
A

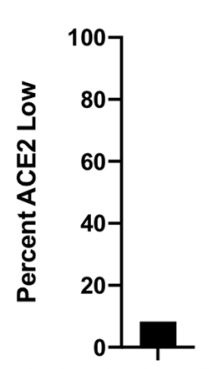

SARS-CoVISARS-CoV-2

High
B

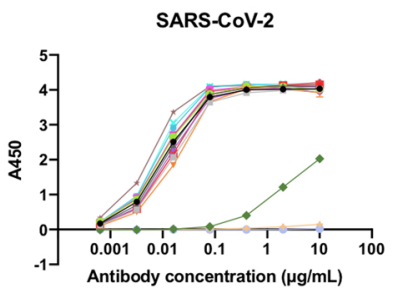

SARS-CoV-2 S1

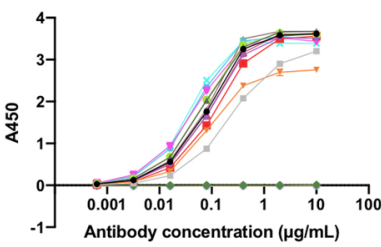

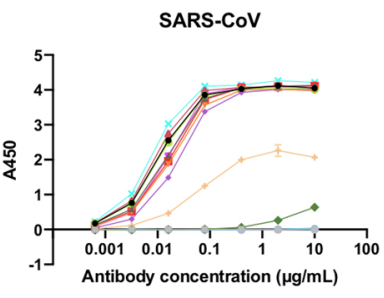

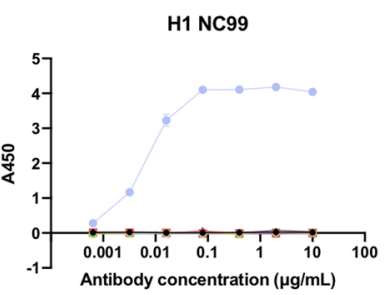

SARS-CoV-2 RBD

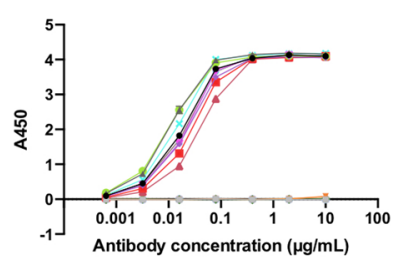

- $5885-1$

- $5885-2$

- 5885-3

$+5885-4$

$\rightarrow 5885-5$

$\rightarrow 5885-6$

$-5885-7$

- $5885-8$

- 5885-9

- 5885-10

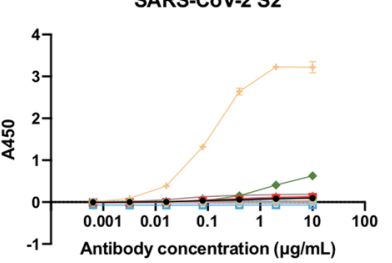

- 5885-11

- 5885-12

- 5885-13

* CR3022

- 3602-1707
SARS-CoV-2

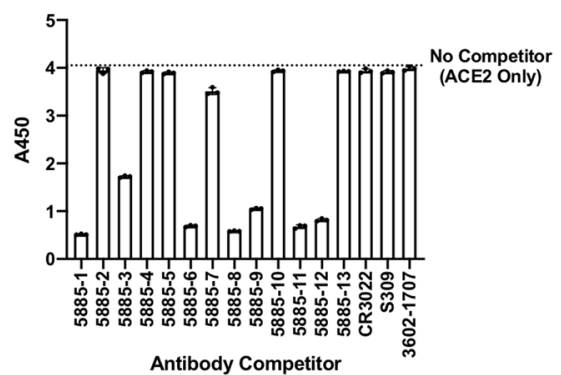

SARS-CoV

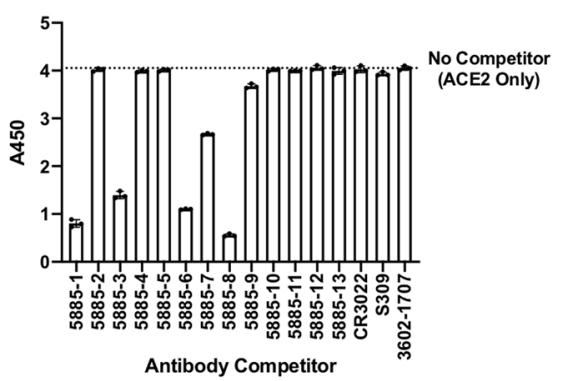

Extended Data Fig. 3 | Characterization of selected cross-reactive antibodies. a. For the IgGs that showed high LIBRA-seq scores ( $>1)$ for both SARS-CoV-2 and SARS-CoV, the percent of cells with low ACE2 scores $(<-1)$ is shown. $\mathbf{b}$. ELISA binding of antibodies to SARS-CoV-2 spike, SARS-CoV spike, influenza hemagglutinin H1 NC99, SARS-CoV-2 S1, SARS-CoV-2 RBD, and SARS-CoV-2 S2. Data are represented as mean \pm SEM of technical duplicates and represent one of at least two independent experiments $(n=2)$. c. ACE2 blocking ELISA. ACE2 binding without competitor is shown as a dotted line. ELISAs were performed at one antibody concentration, and data are represented as mean \pm SEM of technical triplicates and represent one of at least two independent experiments $(n=2)$. 


\section{Reporting Summary}

Nature Research wishes to improve the reproducibility of the work that we publish. This form provides structure for consistency and transparency in reporting. For further information on Nature Research policies, see our Editorial Policies and the Editorial Policy Checklist.

\section{Statistics}

For all statistical analyses, confirm that the following items are present in the figure legend, table legend, main text, or Methods section.

$\mathrm{n} / \mathrm{a}$ Confirmed

$\bigotimes$ The exact sample size $(n)$ for each experimental group/condition, given as a discrete number and unit of measurement

$\bigotimes$ A statement on whether measurements were taken from distinct samples or whether the same sample was measured repeatedly

The statistical test(s) used AND whether they are one- or two-sided

Only common tests should be described solely by name; describe more complex techniques in the Methods section.

\ $\square$ A description of all covariates tested

Х A description of any assumptions or corrections, such as tests of normality and adjustment for multiple comparisons

$\checkmark$ A full description of the statistical parameters including central tendency (e.g. means) or other basic estimates (e.g. regression coefficient)

AND variation (e.g. standard deviation) or associated estimates of uncertainty (e.g. confidence intervals)

For null hypothesis testing, the test statistic (e.g. $F, t, r$ ) with confidence intervals, effect sizes, degrees of freedom and $P$ value noted Give $P$ values as exact values whenever suitable.

Х $\square$ For Bayesian analysis, information on the choice of priors and Markov chain Monte Carlo settings

Х $\square$ For hierarchical and complex designs, identification of the appropriate level for tests and full reporting of outcomes

Х $\square$ Estimates of effect sizes (e.g. Cohen's d, Pearson's $r$ ), indicating how they were calculated

\section{Our web collection on statistics for biologists contains articles on many of the points above.}

\section{Software and code}

Policy information about availability of computer code

Data collection BCR contigs were processed using Cell Ranger 3.1.0 (10X Genomics) using GRCh38 as reference. Antigen barcode libraries were also processed using Cell Ranger 3.1.0 (10X Genomics).

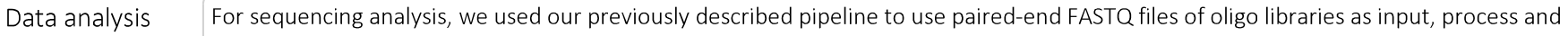
annotate reads for cell barcode, UMI, and antigen barcode, and generate a cell barcode - antigen barcode UMI count matrix (Setliff et al. Cell, 2019)(Shiakolas et al. Cell Reports Medicine, 2021). We also utilized High V-Quest (Alamyar et al. Methods Mol Biol, 2012) and ChangeO (Gupta et al. Bioinformatics, 2015) for further sequence processing.

Additional data in the study was analyzed using GraphPad Prism 8.0.0.

For manuscripts utilizing custom algorithms or software that are central to the research but not yet described in published literature, software must be made available to editors and

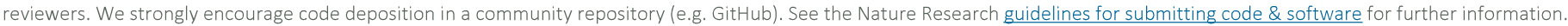

\section{Data}

Policy information about availability of data

All manuscripts must include a data availability statement. This statement should provide the following information, where applicable:

- Accession codes, unique identifiers, or web links for publicly available datasets

- A list of figures that have associated raw data

- A description of any restrictions on data availability

, All unique reagents generated in this study are available from the corresponding author with a completed Materials Transfer Agreement. Sequences for antibodies identified and characterized in this study have been deposited to GenBank (MZ517191-MZ517250, OM001674 


\section{Field-specific reporting}

Please select the one below that is the best fit for your research. If you are not sure, read the appropriate sections before making your selection.

$\bigotimes$ Life sciences $\quad \square$ Behavioural \& social sciences $\quad \square$ Ecological, evolutionary \& environmental sciences

For a reference copy of the document with all sections, see nature.com/documents/nr-reporting-summary-flat.pdf

\section{Life sciences study design}

All studies must disclose on these points even when the disclosure is negative.

Sample size No sample size calculation was performed. This study sought to highlight that potently neutralizing antibodies could be identified using a small number of antibody leads from a single next-generation sequencing experiment. Hundreds of cells were recovered from each of three experiments, and from each, less than a dozen antibodies were selected for expression and validation (as shown in the manuscript).

Data exclusions Prioritized, expressed antibodies (shown in Figure 1) were excluded from characterization analysis if heavy/light chain plasmid sequencing did not match expected sequence or if ELISA binding repeats were not consistent.

Replication All ELISA binding data and ACE2 blocking data were repeated at least twice in technical duplicate. Data are shown as mean +/- SEM Additionally, replicates for neutralization data are shown as mean +/-SD.

Randomization Randomization is not relevant to our study.

Blinding Blinding is not relevant to our study.

\section{Reporting for specific materials, systems and methods}

We require information from authors about some types of materials, experimental systems and methods used in many studies. Here, indicate whether each material, system or method listed is relevant to your study. If you are not sure if a list item applies to your research, read the appropriate section before selecting a response.

Materials \& experimental systems

\begin{tabular}{l|l} 
Methods \\
\hline n/a & Involved in the study \\
$\square$ & $\square$ ChIP-seq \\
$\square$ & $\bigotimes$ Flow cytometry \\
$\square$ & $\square$ MRI-based neuroimaging
\end{tabular}

$\mathrm{n} / \mathrm{a}$ Involved in the study

$\square$ \ Antibodies

$\square \bigotimes$ Eukaryotic cell lines

Х $\square$ Palaeontology and archaeology

Х $\square$ Animals and other organisms

Х $\square$ Human research participants

Х $\square$ Clinical data

Х $\square$ Dual use research of concern

\section{Antibodies}

Antibodies used

Antibodies were used for fluorescence activated cell sorting (FACS): APC-CY'TM7 Mouse Anti-Human CD14 (BD, Cat\#561709; RRID: RRID:AB_10893806), FITC Anti-Human CD3 (Tonbo Biosciences, Cat\#35-0037; RRID:AB_2621662), BV711 Mouse Anti-Human CD19 (BD, Cat\#563036; RRID: AB 2737968), and PE-CYTM5 Mouse Anti-Human IgG (BD, Cat\#551497; RRID: AB 394220).

Antibodies were used in binding and neutralization experiments: CR3022 (Yuan et al. Science 2020), 3602-1707 (Setliff et al. Cell 2019), 46472-4 (S2 ab, Shiakolas et al. Cell Reports Medicine 2021, GenBank: MZ126647, MZ126662) , 46472-6 (NTD ab, Shiakolas et al. Cell Reports Medicine 2021, GenBank: MZ126649, MZ126664), 1F8 (Tang et al. Proceedings of the National Academy of Sciences 2014), DENV-2D22 (James Crowe Jr.), and COV2-2130 (Zost et al., Nature 2020).

Validation

$\mathrm{BD}$ and Tonbo Biosciences perform QC testing and validation on fluorescently labeled antibodies. Citations for other antibodies used are provided above. Additionally, antibodies used in binding and neutralization tests were tested for binding and/or neutralization of previously confirmed/known antigens or viral strains.

\section{Eukaryotic cell lines}

Policy information about cell lines

Cell line source(s)

Freestyle 293F cells (ThermoFisher Scientific, Cat\#A14528)

Expi293F cells (ThermoFisher Scientific, Cat\#A14527) 
Authentication

Mycoplasma contamination

Commonly misidentified lines

(See ICLAC register)
ExpiCHO cells were authenticated using ATCC's Cell Line Authentication Service.

ExpiCHO cell line was routinely tested and were negative for mycoplasma contamination. Vero E6 cells were negative for mycoplasma contamination. Mycoplasma testing of cell lines was performed using a PCR-based mycoplasma detection kit (ATCC, 30-1012K) or a mycoplasma detection colorimetry kit.

None.

\section{Flow Cytometry}

Plots

Confirm that:

The axis labels state the marker and fluorochrome used (e.g. CD4-FITC).

The axis scales are clearly visible. Include numbers along axes only for bottom left plot of group (a 'group' is an analysis of identical markers).

All plots are contour plots with outliers or pseudocolor plots.

A numerical value for number of cells or percentage (with statistics) is provided.

\section{Methodology}

Sample preparation

Instrument

Software

Cell population abundance

Gating strategy
PBMC samples were purchased from Cellero and stained for fluorescence activated cell sorting.

A 4-laser FACSAria III and a 5- Laser FACS Aria III were used for FACS experiments.

Flow cytometry data were analyzed using FlowJo. The sorting data are not shown in the manuscript. Summary data from the post-sorting, single cell sequencing antigen reactivity read out are shown in Figure 1.

After sorting, for experiment 1 there were 23,162 antigen+/IgG+ cells. For experiment 2 , there were 65,578 antigen+/IgG+ cells. For experiment 3, there were 19,133 antigen+/lgG+ cells. These cells were then used for single cell processing and sequencing.

Cells were gated on SSC-A and FSC-A. Then, singlets were isolated by gating on SSC-W/SSC-H and FSC-W/FSC-H. Next, cells were gated for negativity to APC-Cy7 (CD14 and LiveDead marker). These Live/CD14- cells were gated for CD3-FITC negative/ CD19-BV711 positive. Lastly, the cells were gated for antigen-PE positivity and IgG-PE-Cy5 positivity. An FMO control without antigen-PE was also used for gating.

Tick this box to confirm that a figure exemplifying the gating strategy is provided in the Supplementary Information. 